TRANSACTIONS OF THE

AMERICAN MATHEMATICAL SOCIETY

Volume 362, Number 8, August 2010, Pages 4137-4160

S 0002-9947(10)04957-3

Article electronically published on March 24, 2010

\title{
GLOBALIZATION OF TWISTED PARTIAL ACTIONS
}

\author{
M. DOKUCHAEV, R. EXEL, AND J. J. SIMÓN
}

\begin{abstract}
Let $\mathcal{A}$ be a unital ring which is a product of possibly infinitely many indecomposable rings. We establish a criteria for the existence of a globalization for a given twisted partial action of a group on $\mathcal{A}$. If the globalization exists, it is unique up to a certain equivalence relation and, moreover, the crossed product corresponding to the twisted partial action is Morita equivalent to that corresponding to its globalization. For arbitrary unital rings the globalization problem is reduced to an extendibility property of the multipliers involved in the twisted partial action.
\end{abstract}

\section{INTRODUCTION}

The relationship between partial isomorphisms and global ones proved to be relevant in diverse areas of mathematics, such as operator theory, topology, logic, graph theory, differential geometry, group theory (with particular importance for geometrical and combinatorial group theories) and semigroup theory (see [20, [21]). Moreover, it naturally can be found as a significant feature even in the basics of the Galois Theory of fields, or in that of inner product spaces in the form of the Witt Lemma (see [21]). The latter was the origin of the term "Witt property", naming an important situation in which every partial isomorphism lies beneath a global one. Other remarkable related properties which reflect certain "globalization" phenomena are the so-called "HNN-property" and that of "homogeneity" (see 21]).

When a group $G$ is given and a partial isomorphism of a structure is attached to each element of $G$ such that the composition of partial isomorphisms respects the group operation, we speak about a partial action of $G$. The formal definition of this concept was given in [13, and the study of globalizations (also called enveloping actions) of partial group actions was initiated in the Ph.D. Thesis of F. Abadie [1] (see also [2]) and independently by J. Kellendonk and M. Lawson in 20]. Further results on globalizations of partial actions of groups were obtained in [22], [8], [16] and [1]. In particular, in [8], a criterion for the existence of a globalization for a partial group action on a unital ring was given, which was further generalized for partial group actions on left $s$-unital rings in [11, incorporating a new ingredient to the criteria, which is essential for the $s$-unital context. One should notice that the concept of a globalization of a partial action of a group on a $\operatorname{ring} \mathcal{A}$, as treated in [2, [8] and [1] (see also Definition 2.1] below), has a rigid condition that $\mathcal{A}$ is an ideal

Received by the editors February 15, 2008.

2010 Mathematics Subject Classification. Primary 16W50; Secondary 16S35, 16W22.

Key words and phrases. Partial action, twisting, crossed product, globalization.

This work was partially supported by $\mathrm{CNPq}$ of Brazil and Secretaría de Estado de Universidades e Investigación del MEC, España. 
in the ring under the global action. This is imposed in the definition because of the way the restriction works: any global group action on a ring canonically restricts to a partial action on a two-sided ideal. However, it may happen that a given partial action restricts to a subring which is not an ideal, and dropping the considered restriction from the definition one comes to the so-called weak globalizations, on which interesting results have been obtained in [16].

Twisted partial actions of locally compact groups on $C^{*}$-algebras were introduced in [12, serving the general construction of $C^{*}$-crossed products. The power of the notion allows one to prove (see [12, Theorem 7.3]) that any second countable $C^{*}$-algebraic bundle with stable unit fiber can be obtained as a result of the construction. Its generality permits us to characterize important classes of $C^{*}$ algebras as $C^{*}$-crossed products (see [15]). Twisted partial actions of groups on abstract rings and corresponding crossed products were introduced in [9], in which an algebraic analog of the above-mentioned stabilization theorem was also proved. As a recent application of the construction, it was proved in [14 that given a field $F$ with $\operatorname{char} F=0$ and a subnormal subgroup $H \triangleleft N \triangleleft G$, there is a twisted partial action of the factor group $G / N$ on the group algebra $F[N / H]$ such that the Hecke algebra $\mathcal{H}(G, H)$ is isomorphic to the partial crossed product $F[N / H] * G / N$. Separability, semisimplicity and Frobenius properties of crossed products by twisted partial actions were investigated in 4 .

The globalization problem for partial actions on rings is essential for the further study of general crossed products and related topics, in particular, the abovementioned result from [8] turned out to be useful in the Galois Theory of partial actions [10, in M. Ferrero's result [16], and in the series of recent preprints by D. Bagio, W. Cortes, M. Ferrero, J. Lazzarin, H. Marubayashi and A. Paques [3], 5], [6], [7, 17. Thus a solution for the globalization problem for the twisted context seems to be rather desirable, which is our present purpose.

Our paper is organized as follows. After giving some preliminaries in Section 2 , we point out in Section 3 a Morita equivalence result with respect to the partial and global crossed products, which is an immediate consequence of the arguments given in 8 and [11. In Section 4, we reduce the question of the existence of a globalization of a twisted partial action of a group on a unital ring to an extendability property of the multipliers involved. Next we pass to consider twisted partial actions on rings $\mathcal{A}$ which are (finite or infinite) products of indecomposable unital rings, first giving in

Section 5 some preliminary facts on transitive twisted partial actions. In Section 6 we construct a more manageable twisted partial action, equivalent to the given one. The idea is borrowed from the corestriction map in Homological Algebra, which turns out to be adaptable to the non-commutative partial framework. In Section 7 we establish our main existence result, whereas in Section 8 we prove that two globalizations of a given twisted partial action of a group on $\mathcal{A}$ are equivalent in some natural sense from the homological point of view, provided that the rings under the global actions are unital.

\section{SOME PRELIMINARIES}

We remind the reader that the multiplier ring of $\mathcal{M}(\mathcal{A})$ of an associative nonnecessarily unital ring $\mathcal{A}$ is the set

$$
\mathcal{M}(\mathcal{A})=\left\{(R, L) \in \operatorname{End}\left({ }_{\mathcal{A}} \mathcal{A}\right) \times \operatorname{End}\left(\mathcal{A}_{\mathcal{A}}\right):(a R) b=a(L b) \text { for all } a, b \in \mathcal{A}\right\}
$$


with component-wise addition and multiplication (see [8] or [18, 3.12.2] for more details). Here we use the right hand side notation for homomorphisms of left $\mathcal{A}$ modules, whereas for homomorphisms of right modules the usual notation is used. Thus given $R:{ }_{\mathcal{A}} \mathcal{A} \rightarrow_{\mathcal{A}} \mathcal{A}, L: \mathcal{A}_{\mathcal{A}} \rightarrow \mathcal{A}_{\mathcal{A}}$ and $a \in \mathcal{A}$ we write $a \mapsto a R$ and $a \mapsto L a$. For a multiplier $w=(R, L) \in \mathcal{M}(\mathcal{A})$ and an element $a \in \mathcal{A}$ we set $a w=a R$ and $w a=L a$, so that one always has $(a w) b=a(w b)(a, b \in \mathcal{A})$. Given a ring isomorphism $\phi: \mathcal{A} \rightarrow \mathcal{A}^{\prime}$, the map $\mathcal{M}(\mathcal{A}) \ni w \mapsto \phi w \phi^{-1} \in \mathcal{M}\left(\mathcal{A}^{\prime}\right)$ where $\phi w \phi^{-1}=\left(\phi^{-1} R \phi, \phi L \phi^{-1}\right), w=(R, L)$, is an isomorphism of rings.

Definition 2.1. A twisted partial action of a group $G$ on $\mathcal{A}$ is a triple

$$
\alpha=\left(\left\{\mathcal{D}_{g}\right\}_{g \in G},\left\{\alpha_{g}\right\}_{g \in G},\left\{w_{g, h}\right\}_{(g, h) \in G \times G)},\right.
$$

where for each $g \in G, \mathcal{D}_{g}$ is a two-sided ideal in $\mathcal{A}, \alpha_{g}$ is an isomorphism of rings $\mathcal{D}_{g^{-1}} \rightarrow \mathcal{D}_{g}$, and for each $(g, h) \in G \times G, w_{g, h}$ is an invertible element from $\mathcal{M}\left(\mathcal{D}_{g} \cdot \mathcal{D}_{g h}\right)$, satisfying the following postulates, for all $g, h$ and $t$ in $G$ :

(i) $\mathcal{D}_{g}^{2}=\mathcal{D}_{g}, \mathcal{D}_{g} \cdot \mathcal{D}_{h}=\mathcal{D}_{h} \cdot \mathcal{D}_{g}$;

(ii) $\mathcal{D}_{1}=\mathcal{A}$ and $\alpha_{1}$ is the identity map of $\mathcal{A}$;

(iii) $\alpha_{g}\left(\mathcal{D}_{g^{-1}} \cdot \mathcal{D}_{h}\right)=\mathcal{D}_{g} \cdot \mathcal{D}_{g h}$;

(iv) $\alpha_{g} \circ \alpha_{h}(a)=w_{g, h} \alpha_{g h}(a) w_{g, h}^{-1}, \forall a \in \mathcal{D}_{h^{-1}} \cdot \mathcal{D}_{h^{-1} g^{-1}}$;

(v) $w_{1, g}=w_{g, 1}=1$;

(vi) $\alpha_{g}\left(a w_{h, t}\right) w_{g, h t}=\alpha_{g}(a) w_{g, h} w_{g h, t}, \forall a \in \mathcal{D}_{g^{-1}} \cdot \mathcal{D}_{h} \cdot \mathcal{D}_{h t}$.

As is mentioned in [9, it follows from (i) that a finite product of ideals $\mathcal{D}_{g} \cdot \mathcal{D}_{h} \cdot \ldots$ is idempotent, and

$$
\alpha_{g}\left(\mathcal{D}_{g^{-1}} \cdot \mathcal{D}_{h} \cdot \mathcal{D}_{f}\right)=\mathcal{D}_{g} \cdot \mathcal{D}_{g h} \cdot \mathcal{D}_{g f}
$$

for all $g, h, f \in G$, by (iii). Thus all multipliers in (vi) are applicable.

We say that $\alpha$ is global if $\mathcal{D}_{g}=\mathcal{A}$ for all $g \in G$. Observe that given a twisted global action

$$
\beta=\left(\mathcal{B},\left\{\beta_{x}\right\}_{x \in G},\left\{u_{x, y}\right\}_{(x, y) \in G \times G)}\right.
$$

of a group $G$ on a (non-necessarily unital) ring $\mathcal{B}$, one may restrict $\beta$ to a two-sided ideal $\mathcal{A}$ of $\mathcal{B}$ such that $\mathcal{A}$ has $1_{\mathcal{A}}$ as follows. Setting $\mathcal{D}_{x}=\mathcal{A} \cap \beta_{x}(\mathcal{A})=\mathcal{A} \cdot \beta_{x}(\mathcal{A})$, we see that each $\mathcal{D}_{x}$ has 1 which is $1_{\mathcal{A}} \beta_{x}\left(1_{\mathcal{A}}\right)$. Then putting $\alpha_{x}=\left.\beta_{x}\right|_{\mathcal{D}_{x-1}}$, the items (i), (ii), (iii) of Definition 2.1 are satisfied. Furthermore, defining $w_{x, y}=$ $u_{x, y} 1_{\mathcal{A}} \beta_{x}\left(1_{\mathcal{A}}\right) \beta_{x y}\left(1_{\mathcal{A}}\right)$ we have that (iv), (v) and (vi) are also satisfied, so we indeed have obtained a twisted partial action on $\mathcal{A}$.

Definition 2.2. A twisted global action

$$
\beta=\left(\mathcal{B},\left\{\beta_{g}\right\}_{g \in G},\left\{u_{g, h}\right\}_{(g, h) \in G \times G)}\right.
$$

of a group $G$ on an associative (non-necessarily unital) $\operatorname{ring} \mathcal{B}$ is said to be a globalization (or an enveloping action) for the partial action $\alpha$ of $G$ on $\mathcal{A}$ if there exists a monomorphism $\varphi: \mathcal{A} \rightarrow \mathcal{B}$ such that:

(i) $\varphi(\mathcal{A})$ is an ideal in $\mathcal{B}$,

(ii) $\mathcal{B}=\sum_{g \in G} \beta_{g}(\varphi(\mathcal{A}))$,

(iii) $\varphi\left(\mathcal{D}_{g}\right)=\varphi(\mathcal{A}) \cap \beta_{g}(\varphi(\mathcal{A}))$ for any $g \in G$,

(iv) $\varphi \circ \alpha_{g}=\beta_{g} \circ \varphi$ on $\mathcal{D}_{g^{-1}}$ for any $g \in G$,

(v) $\varphi\left(a w_{g, h}\right)=\varphi(a) u_{g, h}, \varphi\left(w_{g, h} a\right)=u_{g, h} \varphi(a)$ for any $g, h \in G$ and $a \in \mathcal{D}_{g} \mathcal{D}_{g h}$. If this is the case, we shall say that $\alpha$ is globalizable. 
Our rings will always be associative, non-necessarily with unity in general. Given a unital ring $\mathcal{A}$, we shall denote by $\mathcal{U}(\mathcal{A})$ the group of invertible elements of $\mathcal{A}$, whereas $1_{\mathcal{A}}$ will stand for the unity of $\mathcal{A}$. If $\beta$ is a globalization for $\alpha$, then $\mathcal{B}$ may have no unity even when $\mathcal{A}$ is a ring with $1_{\mathcal{A}}$; if, however, $\mathcal{B}$ has $1_{\mathcal{B}}$, then we say that $\beta$ is a unital globalization and $\alpha$ is unitally globalizable. The multipliers $w_{x, y}$ of a twisted partial action will also be denoted by $w[x, y]$.

\section{Morita equivalence}

It was proved in 8 , that if $\alpha$ is a (non-twisted) partial action of a group $G$ on a unital ring $\mathcal{A}$ and $\beta$ is a globalization of $\alpha$ with $G$ acting (globally) on $\mathcal{B}$ such that $\mathcal{B}$ has $1_{\mathcal{B}}$, then the partial skew group ring $\mathcal{A} *_{\alpha} G$ is Morita equivalent to the (global) skew group ring $\mathcal{B} *_{\beta} G$. The proof is easily adaptable for the left $s$-unital case, as was shown in [11. We recall that a $\operatorname{ring} \mathcal{A}$ is called left $s$-unital if for any $a \in \mathcal{A}$ there exists an element $e \in \mathcal{A}$ such that $e a=a$. Equivalently, for any finitely many $a_{1}, \ldots, a_{k} \in \mathcal{A}$ there exists an element $e \in \mathcal{A}$ with $e a_{i}=a_{i}, i=1, \ldots, k$ (see [11, Lemma 2.4]). Any left $s$-unital ring is obviously idempotent, and for them the Morita theory of idempotent rings developed in [19] is applicable. We also recall that given a twisted partial action $\alpha$ of $G$ on $\mathcal{A}$, the crossed product $\mathcal{A} *_{\alpha} G$ is the direct sum:

$$
\bigoplus_{g \in G} \mathcal{D}_{g} \delta_{g}
$$

in which the $\delta_{g}$ 's are symbols, and the multiplication is defined by the rule:

$$
\left(a_{g} \delta_{g}\right) \cdot\left(b_{h} \delta_{h}\right)=\alpha_{g}\left(\alpha_{g}^{-1}\left(a_{g}\right) b_{h}\right) w_{g, h} \delta_{g h} .
$$

Here $w_{g, h}$ acts as a right multiplier on $\alpha_{g}\left(\alpha_{g}^{-1}\left(a_{g}\right) b_{h}\right) \in \alpha_{g}\left(\mathcal{D}_{g^{-1}} \cdot \mathcal{D}_{h}\right)=\mathcal{D}_{g} \cdot \mathcal{D}_{g h}$. The associativity of this construction was established in [9].

We point out the next:

Theorem 3.1. Let $\alpha$ be a globalizable twisted partial action of $G$ on a left s-unital ring $\mathcal{A}$ and let $\beta$ be a globalization of $\alpha$ with $G$ acting globally on $\mathcal{B}$. Then the crossed products $\mathcal{A} *_{\alpha} G$ and $\mathcal{B} *_{\beta} G$ are Morita equivalent.

Proof. We observe that the Morita context and the arguments given in [8] with the adaptation for the $s$-unital case in [11] work nicely, and we give only a sketch of the proof.

Since $\mathcal{A}$ is left $s$-unital, so too is $\mathcal{B}$, as a sum of left $s$-unital ideals (see [11, Remark 2.5]). By [11, Theorem 3.1] each $\mathcal{D}_{g}$ is left $s$-unital and consequently, $\mathcal{R}=\mathcal{A} *_{\alpha} G$ and $\mathcal{R}^{\prime}=\mathcal{B} *_{\beta} G$ are also left $s$-unital. We view $\mathcal{R}$ as a subring of $\mathcal{R}^{\prime}$. Consider $M, N \subseteq \mathcal{B} *_{\beta} G$ given by

$$
M=\left\{\sum_{g \in G} c_{g} u_{g}: c_{g} \in \mathcal{A}\right\} \text { and } N=\left\{\sum_{g \in G} c_{g} u_{g}: c_{g} \in \beta_{g}(\mathcal{A})\right\} .
$$

The proofs of Propositions 5.1 and 5.2 of [8] do not use the fact that the ring has 1 and work without essential changes in the twisted context. According to these propositions $M$ is an $\mathcal{R}$ - $\mathcal{R}^{\prime}$ - bimodule and $N$ is an $\mathcal{R}^{\prime}-\mathcal{R}$ - bimodule. While checking this, one should keep in mind that all ideals $\beta_{x}(\mathcal{A})(x \in G)$ are idempotent, and therefore, $u[y, z] \beta_{x}(\mathcal{A})=\beta_{x}(\mathcal{A}) u[y, z]=\beta_{x}(\mathcal{A})$ for any $x, y, z \in G$, as invertible multipliers preserve idempotent ideals. 
Next, let $\tau: M \otimes_{\mathcal{R}^{\prime}} N \rightarrow \mathcal{R}^{\prime}$ and $\tau^{\prime}: N \otimes_{\mathcal{R}} M \rightarrow \mathcal{R}^{\prime}$ be given by $\tau(m \otimes n)=m n$ and $\tau^{\prime}(n \otimes m)=n m$. Obviously $\tau$ is an $\mathcal{R}$-bimodule map and $\tau^{\prime}$ is an $\mathcal{R}^{\prime}$-bimodule map. Similarly as in the proof of Theorem 4.1 of [11] one directly verifies that $\tau\left(M \otimes_{\mathcal{R}^{\prime}} N\right)=\mathcal{R}$ and $\tau^{\prime}\left(N \otimes_{\mathcal{R}} M\right)=\mathcal{R}^{\prime}$.

The notion of the Morita context for idempotent rings is the same as for rings with 1 (see 19); thus we have a Morita context $\left(\mathcal{R}, \mathcal{R}^{\prime}, M, N, \tau, \tau^{\prime}\right)$ with idempotent $R$ and $R^{\prime}$, and surjective $\tau$ and $\tau^{\prime}$. Therefore by [19], the rings $\mathcal{R}$ and $\mathcal{R}^{\prime}$ are Morita equivalent.

\section{REDUCing the GLOBAlization PROBlem to AN EXTENDABILITY PROPERTY OF MULTIPLIERS}

If $\alpha$ is a globalizable twisted partial action of $G$ on a unital ring $\mathcal{A}$, then each $\mathcal{D}_{g}(g \in G)$ is a unital ring, as $\varphi\left(\mathcal{D}_{g}\right)=\varphi(\mathcal{A}) \cap \beta_{g}(\varphi(\mathcal{A}))$. In the non-twisted case the converse is true by [8, Theorem 4.5]. In the twisted case, without imposing restrictions on $\mathcal{A}$, we are able to show in Theorem 4.1 below that the converse is true provided that an extendability property of the partial twisting $w$ is verified (the latter is also a necessary condition). If each $\mathcal{D}_{g}$ is a unital ring whose unity element is denoted by $1_{g}$, then $1_{g}$ is a central idempotent of $\mathcal{A}$ such that $\mathcal{D}_{g}=1_{g} \mathcal{A}$, and for every $g, h \in G$ the ring $\mathcal{D}_{g} \cap \mathcal{D}_{h}=\mathcal{D}_{g} \cdot \mathcal{D}_{h}$ is unital with unity $1_{g} 1_{h}$. Consequently $\mathcal{M}\left(\mathcal{D}_{g} \mathcal{D}_{g h}\right) \cong \mathcal{D}_{g} \mathcal{D}_{g h}$, so that $w_{g, h}$ is an invertible element of $\mathcal{D}_{g} \mathcal{D}_{g h}$ for any $g, h \in G$. Moreover, by (iii) of Definition 2.1

$$
\alpha_{g}\left(1_{g^{-1}} 1_{h}\right)=1_{g} 1_{g h},
$$

for any $g, h \in G$.

Theorem 4.1. Let $\alpha$ be a twisted partial action of a group $G$ on a unital ring $\mathcal{A}$ such that each $\mathcal{D}_{g}(g \in G)$ is a unital ring with unity element $1_{g}$. Then $\alpha$ admits a globalization if and only if for each pair $(g, h) \in G \times G$ there exists an invertible element $\widetilde{w}_{g, h} \in \mathcal{U}(\mathcal{A})$, such that $\widetilde{w}_{g, h} 1_{g} 1_{g h}=w_{g, h}$, and

$$
\alpha_{g}\left(\widetilde{w}_{h, t} 1_{g^{-1}}\right) \widetilde{w}_{g, h t}=1_{g} \widetilde{w}_{g, h} \widetilde{w}_{g h, t},
$$

for any $g, h, t \in G$.

Proof. The "only if" part is obvious by taking $\widetilde{w}_{g, h}=u_{g, h} \cdot 1_{\mathcal{A}}$ for any $g, h \in G$.

For the "if" part let $\mathcal{F}=\mathcal{F}(G, \mathcal{A})$ be the Cartesian product of the copies of $\mathcal{A}$ indexed by the elements of $G$, that is, the ring of all functions from $G$ into $\mathcal{A}$. For convenience of notation $f(g)$ will also be written as $\left.f\right|_{g}(f \in \mathcal{F}, g \in G)$.

For $g \in G$ and $f \in \mathcal{F}$ define $\beta_{g}(f) \in \mathcal{F}$ by the formula:

$$
\left.\beta_{g}(f)\right|_{h}=\widetilde{w}_{h^{-1}, g} f\left(g^{-1} h\right) \widetilde{w}_{h^{-1}, g}^{-1}, \quad h \in G .
$$

Obviously $f \mapsto \beta_{g}(f)$ determines an automorphism $\beta_{g}$ of $\mathcal{F}$. Define $u_{g, h} \in \mathcal{U}(\mathcal{F})$ by setting

$$
\left.u_{g, h}\right|_{t}=\widetilde{w}_{t^{-1}, g} \widetilde{w}_{t^{-1} g, h} \widetilde{w}_{t^{-1}, g h}^{-1}, \quad g, h, t \in G .
$$

For arbitrary $g, h, t, x \in G$ one has that

$$
\begin{aligned}
& \left.\left(\beta_{g}\left(u_{h, t}\right) u_{g, h t}\right)\right|_{x}=\left.\left.\widetilde{w}_{x^{-1}, g} u_{h, t}\right|_{g^{-1} x} \widetilde{w}_{x^{-1}, g}^{-1} u_{g, h t}\right|_{x} \\
& =\left(\widetilde{w}_{x^{-1}, g} \widetilde{w}_{x^{-1} g, h} \widetilde{w}_{x^{-1} g h, t} \widetilde{w}_{x^{-1} g, h t}^{-1} \widetilde{w}_{x^{-1}, g}^{-1}\right)\left(\widetilde{w}_{x^{-1}, g} \widetilde{w}_{x^{-1} g, h t} \widetilde{w}_{x^{-1}, g h t}^{-1}\right) \\
& =\widetilde{w}_{x^{-1}, g} \widetilde{w}_{x^{-1} g, h} \widetilde{w}_{x^{-1} g h, t} \widetilde{w}_{x^{-1}, g h t}^{-1},
\end{aligned}
$$


and

$$
\begin{aligned}
& \left.\left(u_{g, h} u_{g h, t}\right)\right|_{x} \\
& \quad=\left(\widetilde{w}_{x^{-1}, g} \widetilde{w}_{x^{-1} g, h} \widetilde{w}_{x^{-1}, g h}^{-1}\right)\left(\widetilde{w}_{x^{-1}, g h} \widetilde{w}_{x^{-1} g h, t} \widetilde{w}_{x^{-1}, g h t}^{-1}\right) \\
& \quad=\widetilde{w}_{x^{-1}, g} \widetilde{w}_{x^{-1} g, h} \widetilde{w}_{x^{-1} g h, t} \widetilde{w}_{x^{-1}, g h t}^{-1},
\end{aligned}
$$

which shows that

$$
\beta_{g}\left(u_{h, t}\right) u_{g, h t}=u_{g, h} u_{g h, t}
$$

i.e. $u$ satisfies the 2-cocycle equality (vi) of Definition 2.1.

Next for any $f \in \mathcal{F}$ and $g, h, x \in G$ we compute

$$
\begin{aligned}
\left.\left(\beta_{g} \circ \beta_{h}(f)\right)\right|_{x} & =\left.\widetilde{w}_{x^{-1}, g} \beta_{h}(f)\right|_{g^{-1} x} \widetilde{w}_{x^{-1}, g}^{-1} \\
& =\widetilde{w}_{x^{-1}, g} \widetilde{w}_{x^{-1} g, h} f\left(h^{-1} g^{-1} x\right) \widetilde{w}_{x^{-1} g, h}^{-1} \widetilde{w}_{x^{-1}, g}^{-1} .
\end{aligned}
$$

Furthermore,

$$
\begin{aligned}
& \left.\left(u_{g, h} \beta_{g h}(f) u_{g, h}^{-1}\right)\right|_{x} \\
& =\left(\widetilde{w}_{x^{-1}, g} \widetilde{w}_{x^{-1}, h} \widetilde{w}_{x^{-1}, g h}^{-1}\right) \widetilde{w}_{x^{-1}, g h} f\left(h^{-1} g^{-1} x\right) \widetilde{w}_{x^{-1}, g h}^{-1}\left(\widetilde{w}_{x^{-1}, g} \widetilde{w}_{x^{-1} g, h} \widetilde{w}_{x^{-1}, g h}^{-1}\right)^{-1} \\
& =\widetilde{w}_{x^{-1}, g} \widetilde{w}_{x^{-1}, h} f\left(h^{-1} g^{-1} x\right) \widetilde{w}_{x^{-1} g, h}^{-1} \widetilde{w}_{x^{-1}, g}^{-1},
\end{aligned}
$$

and consequently,

$$
\beta_{g} \circ \beta_{h}(f)=u_{g, h} \beta_{g h}(f) u_{g, h}^{-1} ;
$$

i.e. $u$ satisfies (iv) of Definition 2.1. Since trivially $u_{1, g}=u_{g, 1}=1$ for all $g \in G$, we have a twisted global action of $G$ on $\mathcal{F}$.

For any $a \in \mathcal{A}$ the element $a 1_{g}$ belongs to $\mathcal{D}_{g}$ and the formula

$$
\left.\varphi(a)\right|_{g}=\alpha_{g^{-1}}\left(a 1_{g}\right), g \in G,
$$

defines a monomorphism $\varphi: \mathcal{A} \rightarrow \mathcal{F}$.

Let $\mathcal{B}=\sum_{g \in G} \beta_{g}(\varphi(\mathcal{A}))(g \in G)$. Our purpose is to show that the restriction of $\beta$ to $\mathcal{B}$ is a globalization for $\alpha$. Denote this restriction by the same symbol $\beta$. We proceed by checking property (iv) of Definition 2.2.

For $g, h \in G$ and $a \in \mathcal{D}_{g^{-1}}$ we have

$$
\begin{aligned}
& \left.\beta_{g}(\varphi(a))\right|_{h}=\left.\widetilde{w}_{h^{-1}, g} \varphi(a)\right|_{g^{-1} h} \widetilde{w}_{h^{-1}, g}^{-1}=\widetilde{w}_{h^{-1}, g} \alpha_{h^{-1} g}\left(a 1_{g^{-1} h}\right) \widetilde{w}_{h^{-1}, g}^{-1} \\
& \quad=w_{h^{-1}, g} \alpha_{h^{-1} g}\left(a 1_{g^{-1} h}\right) 1_{h^{-1}} 1_{h^{-1} g} w_{h^{-1}, g}^{-1}=w_{h^{-1}, g} \alpha_{h^{-1} g}\left(a 1_{g^{-1}} 1_{g^{-1} h}\right) w_{h^{-1}, g}^{-1},
\end{aligned}
$$

in view of (11) and the equalities $a=a 1_{g^{-1}}, \widetilde{w}_{h^{-1}, g} 1_{h^{-1}} 1_{h^{-1} g}=w_{h^{-1}, g}$. On the other hand,

$$
\left.\varphi\left(\alpha_{g}(a)\right)\right|_{h}=\alpha_{h^{-1}}\left(\alpha_{g}(a) 1_{h}\right)=\alpha_{h^{-1}}\left(\alpha_{g}(a) 1_{g} 1_{h}\right)=\alpha_{h^{-1}}\left(\alpha_{g}\left(a 1_{g^{-1}} 1_{g^{-1} h}\right)\right),
$$

using again (11). Now the equality $\left.\beta_{g}(\varphi(a))\right|_{h}=\left.\varphi\left(\alpha_{g}(a)\right)\right|_{h}$ follows by (iv) of Definition 2.1, and (iv) of Definition 2.2 is proved.

Next we show that

$$
\varphi\left(\mathcal{D}_{g}\right)=\varphi(\mathcal{A}) \cap \beta_{g}(\varphi(\mathcal{A})),
$$

for all $g \in G$. An element from the right hand side can be written as $\varphi(a)=\beta_{g}(\varphi(b))$ for some $a, b \in \mathcal{A}$. Then for each $h \in G$ the equality $\left.\varphi(a)\right|_{h}=\left.\beta_{g}(\varphi(b))\right|_{h}$ means that

$$
\alpha_{h^{-1}}\left(a 1_{h}\right)=\widetilde{w}_{h^{-1}, g} \alpha_{h^{-1} g}\left(b 1_{g^{-1} h}\right) \widetilde{w}_{h^{-1}, g}^{-1}
$$


Taking $h=1$ this gives $a=\alpha_{g}\left(b 1_{g^{-1}}\right) \in \mathcal{D}_{g}$, as $\widetilde{w}_{1, g}=1$, and, consequently, $\varphi\left(\mathcal{D}_{g}\right) \supseteq \varphi(\mathcal{A}) \cap \beta_{g}(\varphi(\mathcal{A}))$. For the reverse inclusion, given an arbitrary $a \in \mathcal{D}_{g}$, we see using (11) and (iv) of Definition 2.1 that

$$
\begin{aligned}
& \left.\beta_{g}\left(\varphi\left(\alpha_{g}^{-1}(a)\right)\right)\right|_{h}=\widetilde{w}_{h^{-1}, g} \alpha_{h^{-1} g}\left(\alpha_{g}^{-1}(a) 1_{g^{-1} h}\right) \widetilde{w}_{h^{-1}, g}^{-1} \\
& \quad=\alpha_{h^{-1}} \circ \alpha_{g}\left(\alpha_{g}^{-1}(a) 1_{g^{-1}} 1_{g^{-1} h}\right)=\alpha_{h^{-1}}\left(a \alpha_{g}\left(1_{g^{-1}} 1_{g^{-1} h}\right)\right)=\alpha_{h^{-1}}\left(a 1_{h}\right)=\left.\varphi(a)\right|_{h},
\end{aligned}
$$

for all $g, h \in G$. This yields that $\varphi(a)=\beta_{g}\left(\varphi\left(\alpha_{g}^{-1}(a)\right)\right)$ for any $g \in G$, and $\varphi\left(\mathcal{D}_{g}\right) \subseteq$ $\varphi(\mathcal{A}) \cap \beta_{g}(\varphi(\mathcal{A}))$. Hence (4) follows and condition (iii) of Definition 2.2 is also satisfied.

Next we check that $\varphi(\mathcal{A})$ is an ideal in $\mathcal{B}$. To see this, it is enough to show that $\beta_{g}(\varphi(a)) \cdot \varphi(b), \varphi(b) \cdot \beta_{g}(\varphi(a)) \in \varphi(\mathcal{A})$ for all $g \in G$ and $a, b \in \mathcal{A}$. For $h \in G$, using again (11) and (iv) of Definition 2.1] we have

$$
\begin{aligned}
& \left.\left.\beta_{g}(\varphi(a))\right|_{h} \cdot \varphi(b)\right|_{h}=\left.\left.\widetilde{w}_{h^{-1}, g} \varphi(a)\right|_{g^{-1} h} \widetilde{w}_{h^{-1}, g}^{-1} \cdot \varphi(b)\right|_{h} \\
& =\widetilde{w}_{h^{-1}, g} \alpha_{h^{-1} g}\left(a 1_{g^{-1} h}\right) \widetilde{w}_{h^{-1}, g}^{-1} \cdot \alpha_{h^{-1}}\left(b 1_{h}\right)=\alpha_{h^{-1}}\left(\alpha_{g}\left(a 1_{g^{-1}}\right) 1_{h}\right) \cdot \alpha_{h^{-1}}\left(b 1_{h}\right) \\
& =\alpha_{h^{-1}}\left(\alpha_{g}\left(a 1_{g^{-1}}\right) b 1_{h}\right)=\left.\varphi\left(\alpha_{g}\left(a 1_{g^{-1}}\right) b\right)\right|_{h} .
\end{aligned}
$$

Thus

$$
\beta_{g}(\varphi(a)) \cdot \varphi(b)=\varphi\left(\alpha_{g}\left(a 1_{g^{-1}}\right) b\right) \in \varphi(\mathcal{A})
$$

and similarly

$$
\varphi(b) \cdot \beta_{g}(\varphi(a))=\varphi\left(b \alpha_{g}\left(a 1_{g^{-1}}\right)\right) \in \varphi(\mathcal{A}),
$$

as desired.

We show next that our $u$ satisfies (v) of Definition 2.2. For any $g, h, t \in G$, by (vi) of Definition 2.1] we have

$$
\begin{aligned}
& \left.\varphi\left(w_{g, h}\right)\right|_{t}=\alpha_{t^{-1}}\left(w_{g, h} 1_{t}\right)=w_{t^{-1}, g} w_{t^{-1} g, h} w_{t^{-1}, g h}^{-1} \\
& \quad=1_{t^{-1}} 1_{t^{-1} g} 1_{t^{-1} g h} \widetilde{w}_{t^{-1}, g} \widetilde{w}_{t^{-1} g, h} \widetilde{w}_{t^{-1}, g h}=\left.\left(\varphi\left(1_{g} 1_{g h}\right) u_{g, h}\right)\right|_{t},
\end{aligned}
$$

which gives

$$
\varphi\left(w_{g, h}\right)=\varphi\left(1_{g} 1_{g h}\right) u_{g, h}
$$

The latter equality readily implies $\varphi\left(a w_{g, h}\right)=\varphi(a) u_{g, h}$ for any $a \in \mathcal{D}_{g} \mathcal{D}_{g h}$, and the second equality in (v) of Definition 2.2 follows similarly.

It remains to prove that

$$
u_{g, h} \mathcal{B}=\mathcal{B}=\mathcal{B} u_{g, h}
$$

for all $g, h \in G$.

First observe that

$$
u_{g, h} \varphi(a)=\varphi\left(\widetilde{w}_{g, h} a\right)
$$

for all $g, h \in G$ and $a \in \mathcal{A}$. Indeed, for any $t \in G$, using (2), one has

$$
\begin{aligned}
\left.\left(u_{g, h} \varphi(a)\right)\right|_{t} & =\widetilde{w}_{t^{-1}, g} \widetilde{w}_{t^{-1} g, h} \widetilde{w}_{t^{-1}, g h}^{-1} \alpha_{t^{-1}}\left(a 1_{t}\right) \\
& =\alpha_{t^{-1}}\left(w_{g, h} 1_{t}\right) \alpha_{t^{-1}}\left(a 1_{t}\right)=\left.\varphi\left(\widetilde{w}_{g, h} a\right)\right|_{t},
\end{aligned}
$$

as claimed. It follows that

$$
u_{g, h} \varphi(a) \in \varphi(\mathcal{A}),
$$

for all $g, h \in G$. 
It is analogously seen that $u_{g, h}^{-1} \varphi(a)=\varphi\left(\widetilde{w}_{g, h}^{-1} a\right)$, so that

$$
u_{g, h}^{-1} \varphi(a) \in \varphi(\mathcal{A})
$$

for all $g, h \in G$.

Next we work with the product $\beta_{t}^{-1}\left(u_{g, h}\right) \varphi(a)$ with arbitrary $g, h, t \in G$ and $a \in \mathcal{A}$. Note that in view of (3),

$$
\beta_{t}^{-1}(f)=u_{t^{-1}, t}^{-1} \beta_{t^{-1}}(f) u_{t^{-1}, t}
$$

for any $f \in \mathcal{F}$. Consequently, applying (2), we have

$$
\begin{aligned}
\beta_{t}^{-1}\left(u_{g, h}\right) \varphi(a) & =u_{t^{-1}, t}^{-1} \beta_{t^{-1}}\left(u_{g, h}\right) u_{t^{-1}, t} \varphi(a) \\
& =u_{t^{-1}, t}^{-1} u_{t^{-1}, g} u_{t^{-1} g, h} u_{t^{-1}, g h}^{-1} u_{t^{-1}, t} \varphi(a),
\end{aligned}
$$

which is contained in $\varphi(\mathcal{A})$ by (6) and (7). This yields that $\beta_{t}^{-1}\left(u_{g, h}\right) \varphi(\mathcal{A}) \subseteq \varphi(\mathcal{A})$, and applying $\beta_{t}$ we obtain that

$$
u_{g, h} \beta_{t}(\varphi(\mathcal{A})) \subseteq \beta_{t}(\varphi(\mathcal{A})),
$$

for all $g, h, t \in G$. Hence $u_{g, h} \mathcal{B} \subseteq \mathcal{B}$, and one similarly shows that $u_{g, h}^{-1} \mathcal{B} \subseteq \mathcal{B}$. An analogous argument gives $\mathcal{B} u_{g, h}, \mathcal{B} u_{g, h}^{-1} \subseteq \mathcal{B}$, and (5) follows.

\section{SOME REMARKS ON TRANSITIVE TWISTED PARTIAL ACTIONS}

In this section we start our treatment of twisted partial actions on products of indecomposable rings by making some preliminary remarks. Let

$$
\mathcal{A}=\prod_{\lambda \in \Lambda} \mathcal{R}_{\lambda}
$$

where each $\mathcal{R}_{\lambda}$ is an indecomposable unital ring and $\Lambda$ is some non-necessarily finite index set. It is directly verified that a decomposition of $\mathcal{A}$ into a product of indecomposable factors (blocks of $\mathcal{A}$ ) is unique up to a permutation of indices. Clearly, each ideal of $\mathcal{A}$ which is a direct factor, i.e. is generated by an idempotent which is central in $\mathcal{A}$, has to be a product of some blocks. Thus, if a twisted partial action $\alpha$ of a group $G$ on $\mathcal{A}$ is given such that each $\mathcal{D}_{g}$ is a unital ring, then for every $g \in G$ the ideal $\mathcal{D}_{g}$ is a product of some $\mathcal{R}_{\lambda}$ 's. It follows that $\alpha$ permutes the indecomposable factors in the sense that if $g \in G$ and $\lambda \in \Lambda$ are such that $\mathcal{R}_{\lambda} \subseteq \mathcal{D}_{g^{-1}}$, then $\alpha_{g}\left(\mathcal{R}_{\lambda}\right)=\mathcal{R}_{\lambda^{\prime}}$ for some $\lambda^{\prime} \in \Lambda$. Note also that since each $\mathcal{D}_{g}$ is unital, $w_{g, h} \in \mathcal{U}\left(\mathcal{D}_{g} \mathcal{D}_{g h}\right)$ for any $g, h \in G$, and hence $w_{g, h} \mathcal{R}_{\lambda}$ is either 0 or $\mathcal{R}_{\lambda}$ for any $\lambda \in \Lambda$, and similarly for $\mathcal{R}_{\lambda} w_{g, h}$. We shall say that $\alpha$ is transitive if for any $\lambda, \lambda^{\prime} \in \Lambda$ there exists $g \in G$ such that $\mathcal{R}_{\lambda} \subseteq \mathcal{D}_{g^{-1}}$ and $\alpha_{g}\left(\mathcal{R}_{\lambda}\right)=\mathcal{R}_{\lambda^{\prime}}$. Fix an arbitrary block from (8) which shall be denoted by $\mathcal{R}_{1}$. It is readily verified that $\alpha$ is transitive if and only if for any $\lambda \in \Lambda$ there exists $g \in G$ such that $\mathcal{R}_{1} \subseteq \mathcal{D}_{g^{-1}}$ and $\alpha_{g}\left(\mathcal{R}_{1}\right)=\mathcal{R}_{\lambda}$.

Suppose that $\alpha$ is transitive and set

$$
H=\operatorname{St}_{G}\left(\mathcal{R}_{1}\right)=\left\{g \in G: \mathcal{R}_{1} \subseteq \mathcal{D}_{g^{-1}}, \alpha_{g}\left(\mathcal{R}_{1}\right)=\mathcal{R}_{1}\right\},
$$

the stabilizer of $\mathcal{R}_{1}$ in $G$, and let $\Lambda^{\prime} \ni 1$ be a left transversal of $H$ in $G$, i.e. $G=\bigcup_{g \in \Lambda^{\prime}} g H$, a disjoint union. One may evidently assume that $\Lambda \ni 1$ is a subset 
of $\Lambda^{\prime}$ so that $\mathcal{R}_{1} \subseteq \mathcal{D}_{g^{-1}}$ for all $g \in \Lambda$ and (8) can be rewritten as

$$
\mathcal{A}=\prod_{g \in \Lambda} \mathcal{R}_{g}
$$

where $\mathcal{R}_{g}=\alpha_{g}\left(\mathcal{R}_{1}\right)$.

For $x \in G$ denote by $\bar{x}$ the element of $\Lambda^{\prime}$ with $\bar{x} H=x H$. Keeping our notation we shall use the next easy properties.

Lemma 5.1. For $g, x \in G$ we have:

(i) $g \in \Lambda^{\prime}, \mathcal{R}_{1} \subseteq \mathcal{D}_{g^{-1}} \Longleftrightarrow g \in \Lambda$;

(ii) $g, \overline{x g} \in \Lambda \Longleftrightarrow \mathcal{R}_{g} \subseteq \mathcal{D}_{x^{-1}}$, and if this holds, then $\alpha_{x}\left(\mathcal{R}_{g}\right)=\mathcal{R}_{\overline{x g}}$;

(iii) $g \in \Lambda^{\prime}, \mathcal{R}_{\overline{x^{-1} g}} \subseteq \mathcal{D}_{x^{-1}} \Longrightarrow g \in \Lambda$.

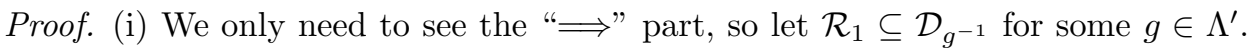
Then $\alpha_{g}\left(\mathcal{R}_{1}\right)$ must be a block of $\mathcal{A}$, so it equals $\mathcal{R}_{t}$ for some $t \in \Lambda$. Hence

$$
\begin{aligned}
\mathcal{R}_{1} & =\alpha_{g}^{-1} \circ \alpha_{t}\left(\mathcal{R}_{1}\right)=w_{g^{-1}, g}^{-1} \alpha_{g^{-1}} \circ \alpha_{t}\left(\mathcal{R}_{1}\right) w_{g^{-1}, g} \\
& =w_{g^{-1}, g}^{-1} w_{g^{-1}, t} \alpha_{g^{-1} t}\left(\mathcal{R}_{1}\right) w_{g^{-1}, t}^{-1} w_{g^{-1}, g}=\alpha_{g^{-1}}\left(\mathcal{R}_{1}\right) .
\end{aligned}
$$

Consequently, $g^{-1} t \in H$, and $g=t \in \Lambda$.

(ii) Let $g, \overline{x g} \in \Lambda$. Then $\mathcal{R}_{1} \subseteq \mathcal{D}_{(\overline{x g})^{-1}} \cap \mathcal{D}_{g^{-1}}$, and since $(\overline{x g})^{-1} x g \in H$, one has that $\alpha_{\overline{x g}} \circ \alpha_{(\overline{x g})^{-1} x g}$ is applicable to $\mathcal{R}_{1}$ and by Definition 2.1 so too is $\alpha_{x g}=$ $\alpha_{\overline{x g} \cdot(\overline{x g})^{-1} x g}$. Thus $\mathcal{R}_{1} \subseteq \mathcal{D}_{(x g)^{-1}}$ and using again Definition 2.1] we see that $\mathcal{R}_{g}=$ $\alpha_{g}\left(\mathcal{R}_{1}\right) \subseteq \alpha_{g}\left(\mathcal{D}_{(x g)^{-1}} \cap \mathcal{D}_{g^{-1}}\right)=\mathcal{D}_{x^{-1}} \cap \mathcal{D}_{g}$, so that $\alpha_{x}$ is applicable to $\mathcal{R}_{g}$. Moreover,

$$
\begin{aligned}
\alpha_{x}\left(\mathcal{R}_{g}\right) & =w_{x, g} \alpha_{x g}\left(\mathcal{R}_{1}\right) w_{x, g}^{-1} \\
& =w_{x, g} w_{\overline{x g},(\overline{x g})^{-1} x g}^{-1} \alpha_{\overline{x g}} \circ \alpha_{(\overline{x g})^{-1} x g}\left(\mathcal{R}_{1}\right) w_{\overline{x g},(\overline{x g})^{-1} x g} w_{x, g}^{-1} \\
& =w_{x, g} w_{\overline{x g},(\overline{x g})^{-1} x g} \alpha_{\overline{x g}}\left(\mathcal{R}_{1}\right) w_{\overline{x g},(\overline{x g})^{-1} x g} w_{x, g}^{-1} \subseteq \mathcal{R}_{\overline{x g}},
\end{aligned}
$$

and consequently $\alpha_{x}\left(\mathcal{R}_{g}\right)=\mathcal{R}_{\overline{x g}}$, as $\alpha_{x}\left(\mathcal{R}_{g}\right)$ must be a block.

Conversely,

$$
\begin{aligned}
& \mathcal{R}_{g} \subseteq \mathcal{D}_{x^{-1}} \Longrightarrow g \in \Lambda, \mathcal{R}_{g} \subseteq \mathcal{D}_{x^{-1}} \cap \mathcal{D}_{g}=\mathcal{D}_{x^{-1}} \mathcal{D}_{g} \Longrightarrow \\
& \mathcal{R}_{1}=\alpha_{g}^{-1}\left(\mathcal{R}_{g}\right) \subseteq \alpha_{g}^{-1}\left(\mathcal{D}_{x^{-1}} \mathcal{D}_{g}\right) \subseteq \mathcal{D}_{g^{-1} x^{-1}} \Longrightarrow \mathcal{R}_{1} \subseteq \mathcal{D}_{g^{-1} x^{-1}} \cap \mathcal{D}_{g^{-1} x^{-1} \overline{x g}} \Longrightarrow \\
& \mathcal{R}_{1}=\alpha_{(\overline{x g})^{-1} x g}\left(\mathcal{R}_{1}\right) \subseteq \alpha_{(\overline{x g})^{-1} x g}\left(\mathcal{D}_{g^{-1} x^{-1}} \cap \mathcal{D}_{g^{-1} x^{-1} \overline{x g}}\right) \subseteq \mathcal{D}_{(\overline{x g})^{-1}}
\end{aligned}
$$

which gives $\overline{x g} \in \Lambda$. $g \in \Lambda$.

(iii) If $\mathcal{R}_{\overline{x^{-1} g}} \subseteq \mathcal{D}_{x^{-1}}$ then by (ii) we have that $\alpha_{x}\left(\mathcal{R}_{\overline{x^{-1} g}}\right)=\left(\mathcal{R}_{\overline{x x^{-1} g}}\right)=\mathcal{R}_{g}$, i.e.

Note that taking $g=1$ in (ii) of Lemma 5.1, we have that

$$
\mathcal{R}_{1} \subseteq \mathcal{D}_{x^{-1}} \Longleftrightarrow \mathcal{R}_{1} \subseteq \mathcal{D}_{\bar{x}^{-1}},
$$

for any $x \in G$.

Given $g \in \Lambda$, we denote by $\operatorname{pr}_{g}: \mathcal{A} \rightarrow \mathcal{R}_{g}$ the projection map; the element $\operatorname{pr}_{g}(a)$ shall be called the $g$-entry of $a$. Using this notation one may write

$$
a=\prod_{g \in \Lambda} \operatorname{pr}_{g}(a)
$$


for any $a \in \mathcal{A}$. We shall work with multiplicative maps $\theta_{x}: \mathcal{A} \rightarrow \mathcal{A},(x \in G)$, defined as follows: for arbitrary $a \in \mathcal{A}$ let $\theta_{x}(a)=1_{\mathcal{A}}$ if $\overline{x^{-1}} \notin \Lambda$, and if $\overline{x^{-1}} \in \Lambda$, i.e. $\mathcal{R}_{1} \subseteq \mathcal{D}_{x}$, then

$$
\operatorname{pr}_{g} \theta_{x}(a)=\left\{\begin{array}{cl}
1_{\mathcal{R}_{g}}, & \text { if } g \neq \overline{x-1} \\
\operatorname{pr}_{g} \alpha_{x}^{-1}\left(\operatorname{pr}_{1} a\right), & \text { otherwise. }
\end{array}\right.
$$

We shall frequently use the next obvious equality:

$$
\theta_{x}(a)=\theta_{x}\left(a 1_{h}\right)
$$

for any $x \in G, a \in \mathcal{A}$ and $h \in H=\operatorname{St}_{G}\left(\mathcal{R}_{1}\right)$. We also need the following properties.

Lemma 5.2. For any $x \in G$ and $g \in \Lambda^{\prime}$ we have

(i) $1_{x} \theta_{g^{-1}}\left(a 1_{g^{-1} x}\right)=1_{x} \theta_{g^{-1}}(a)$, for all $a \in \mathcal{A}$;

(ii) $1_{x} \theta_{g^{-1}} \circ \alpha_{g^{-1}} \circ \alpha_{x} \circ \alpha_{\left(\overline{x^{-1} g}\right)^{-1}}^{-1}(a)=\alpha_{x}\left(1_{x^{-1}} \theta_{\left(\overline{x^{-1} g}\right)^{-1}}(a)\right)$, for all $a \in$ $\mathcal{D}_{\left(\overline{x^{-1} g}\right)^{-1} x^{-1}} \cap \mathcal{D}_{\left(\overline{x^{-1} g}\right)^{-1}} \cap \mathcal{D}_{\left(\overline{x^{-1} g}\right)^{-1} x^{-1} g}$.

Proof. (i) Since $\theta_{g^{-1}}$ is multiplicative, it is enough to check that $1_{x} \theta_{g^{-1}}\left(1_{g^{-1}}\right)=1_{x}$. If $g \notin \Lambda$, then $\theta_{g^{-1}}\left(1_{g^{-1} x}\right)=1_{\mathcal{A}}$, and our equality is clearly satisfied. Moreover, the equality $\theta_{g^{-1}}\left(1_{g^{-1} x}\right)=1_{\mathcal{A}}$ also holds if $g \in \Lambda$ and $\mathcal{R}_{1} \subseteq \mathcal{D}_{g^{-1} x}$. If $g \in \Lambda$ and $\mathcal{R}_{1} \nsubseteq \mathcal{D}_{g^{-1} x}$, then the $g$-entry of $\theta_{g^{-1}}\left(1_{g^{-1} x}\right)$ is 0 and all other entries are 1's. By (10) and Lemma 5.1] $\mathcal{R}_{g} \nsubseteq \mathcal{D}_{x}$, and consequently $1_{x} \theta_{g^{-1}}\left(1_{g^{-1} x}\right)=1_{x}$ holds also in this case.

(ii) Note that $\mathcal{D}_{\left(\overline{x^{-1} g}\right)^{-1} x^{-1}} \cap \mathcal{D}_{\left(\overline{x^{-1} g}\right)^{-1}} \cap \mathcal{D}_{\left(\overline{x^{-1} g}\right)^{-1} x^{-1} g}$ is the set of all $a \in \mathcal{A}$ for which $\alpha_{g^{-1}} \circ \alpha_{x} \circ \alpha_{\left(\overline{x^{-1} g}\right)^{-1}}^{-1}$ is applicable. Denote by $a_{1}$ the left hand side of the equality in (ii) and by $a_{2}$ its right hand side. It is easy to see that if $g \notin \Lambda$ or $\overline{x^{-1} g} \notin \Lambda$, then $a_{1}=1_{x}=a_{2}$, so suppose that $g, \overline{x^{-1} g} \in \Lambda$. Evidently $a_{1}, a_{2} \in \mathcal{D}_{x}$ and by the definition of the $\theta$ 's, for any $t \in \Lambda$ with $t \neq g$ and $\mathcal{R}_{t} \subseteq \mathcal{D}_{x}$, we have $\operatorname{pr}_{t}\left(a_{1}\right)=1_{\mathcal{R}_{t}}=\operatorname{pr}_{t}\left(a_{2}\right)$. Furthermore, by Lemma 5.1, $\mathcal{R}_{g} \subseteq \mathcal{D}_{x}$ and

$$
\begin{aligned}
& \operatorname{pr}_{g}\left(a_{1}\right)=\operatorname{pr}_{g}\left(\theta_{g^{-1}} \circ \alpha_{g^{-1}} \circ \alpha_{x} \circ \alpha_{\left(\overline{x^{-1} g}\right)^{-1}}^{-1}(a)\right)=\operatorname{pr}_{g}\left(\alpha_{x} \circ \alpha_{\left(\overline{x^{-1} g}\right)^{-1}}^{-1}(a)\right) \\
& =\operatorname{pr}_{g}\left(\alpha_{x}\left(1_{x^{-1}} \theta_{\left(\overline{x^{-1} g}\right)^{-1}}(a)\right)\right)=\operatorname{pr}_{g}\left(a_{2}\right),
\end{aligned}
$$

and consequently $a_{1}=a_{2}$.

\section{Going to An Equivalent twisted partial aCtion}

In this section we adapt to the partial and non-commutative context the idea of the corestriction from Homological Algebra in order to produce a more manageable twisted partial action, equivalent to the initial one. We give the following:

Definition 6.1. Two twisted partial actions of $G$ on a $\operatorname{ring} \mathcal{A}$,

$$
\alpha=\left(\left\{\mathcal{D}_{x}\right\}_{x \in G},\left\{\alpha_{x}\right\}_{x \in G},\left\{w_{x, y}\right\}_{(x, y) \in G \times G}\right)
$$

and

$$
\alpha^{\prime}=\left(\left\{\mathcal{D}_{x}\right\}_{x \in G},\left\{\alpha_{x}^{\prime}\right\}_{x \in G},\left\{w_{x, y}^{\prime}\right\}_{(x, y) \in G \times G}\right),
$$

such that each $\mathcal{D}_{x}(x \in G)$ is a unital ring with unity $1_{x}$, shall be called equivalent if there exists a function

$$
G \ni x \mapsto \varepsilon_{x} \in \mathcal{U}\left(\mathcal{D}_{x}\right) \subseteq \mathcal{A}
$$


such that for all $x, y \in G$ and $a \in \mathcal{D}_{x^{-1}}$ we have

$$
\alpha_{x}^{\prime}(a)=\varepsilon_{x} \alpha_{x}(a) \varepsilon_{x}^{-1}
$$

and

$$
w_{x, y}^{\prime}=\varepsilon_{x} \alpha_{x}\left(\varepsilon_{y} 1_{x^{-1}}\right) w_{x, y} \varepsilon_{x y}^{-1} .
$$

Observe that in the above definition the twisted partial actions have common domains $\mathcal{D}_{x}$. It is readily checked that the definition is correct; i.e. our relation is reflexive, symmetric and transitive.

Lemma 6.2. Let

$$
\alpha=\left(\left\{\mathcal{D}_{x}\right\}_{x \in G},\left\{\alpha_{x}\right\}_{x \in G},\left\{w_{x, y}\right\}_{(x, y) \in G \times G)}\right.
$$

be a twisted partial action of $G$ on $\mathcal{A}$ such that each $\mathcal{D}_{x}(x \in G)$ is a unital ring with unity $1_{x}$. Given a function

$$
G \ni x \mapsto \varepsilon_{x} \in \mathcal{U}\left(\mathcal{D}_{x}\right) \subseteq \mathcal{A},
$$

define $\alpha_{x}^{\prime}(x \in G)$ by (12) and $w_{x, y}(x, y \in G)$ by (13). Then

$$
\alpha^{\prime}=\left(\left\{\mathcal{D}_{x}\right\}_{x \in G},\left\{\alpha_{x}^{\prime}\right\}_{x \in G},\left\{w_{x, y}^{\prime}\right\}_{(x, y) \in G \times G}\right)
$$

is a twisted partial action of $G$ on $\mathcal{A}$.

Proof. The items (i), (ii), (iii) and (v) of Definition 2.1 are immediate, and the verification of (iv) is straightforward. As to (vi), for arbitrary $x, y, z \in G$ we have

$$
\begin{aligned}
& \alpha_{x}^{\prime}\left(1_{x^{-1}} w_{y, z}^{\prime}\right) w_{x, y z}^{\prime}=\varepsilon_{x} \alpha_{x}\left(1_{x^{-1} \varepsilon_{y}} \alpha_{y}\left(\varepsilon_{z} 1_{y^{-1}}\right) w_{y, z} \varepsilon_{y z}^{-1}\right) \varepsilon_{x}^{-1} \varepsilon_{x} \alpha_{x}\left(\varepsilon_{y z} 1_{x^{-1}}\right) w_{x, y z} \varepsilon_{x y z}^{-1} \\
& =\varepsilon_{x} \alpha_{x}\left(1_{x^{-1}} \varepsilon_{y}\right)\left(\alpha_{x} \circ \alpha_{y}\left(\varepsilon_{z} 1_{y^{-1} x^{-1}} 1_{y^{-1}}\right)\right) w_{x, y} w_{x y, z} \varepsilon_{x y z}^{-1},
\end{aligned}
$$

by cancelling the $\varepsilon$ 's, and using the 2-cocycle equality for the w's and (1). Now composing $\alpha_{x} \circ \alpha_{y}$ according to (iv) of Definition 2.1, we easily come to $1_{x} w_{x, y}^{\prime} w_{x y, z}^{\prime}$, as claimed.

In what follows in this section we assume all notation from the previous one, in particular, that $\mathcal{A}$ is a product of blocks as in (9), and $\alpha$ is a transitive twisted partial action of $G$ on $\mathcal{A}$. We remind the reader that we shall write the element $w_{x, y}$ also as $w[x, y]$, the standard notation used in Homological Algebra for 2-cocycles. Since each $\mathcal{D}_{x}$ has unity $1_{x}$, we can rewrite the 2 -cocycle equality (vi) from Definition 2.1 as

$$
\alpha_{x}\left(1_{x^{-1}} w[y, z]\right) w[x, y z]=w[x, y] w[x y, z], \forall x, y, z \in G
$$

Set

$$
w^{\prime}[x, y]=1_{x} 1_{x y} \prod_{g \in \Lambda} \theta_{g^{-1}}\left(w\left[g^{-1} x \cdot \overline{x^{-1} g},\left(\overline{x^{-1} g}\right)^{-1} \cdot y \cdot \overline{y^{-1} x^{-1} g}\right]\right) .
$$

Since $\mathcal{R}_{1} \subseteq \mathcal{D}_{h}$ for any $h \in H=\operatorname{St}_{G}\left(\mathcal{R}_{1}\right)$, and

$$
w\left[g^{-1} x \cdot \overline{x^{-1} g},\left(\overline{x^{-1} g}\right)^{-1} \cdot y \cdot \overline{y^{-1} x^{-1} g}\right] \in \mathcal{U}\left(\mathcal{D}_{g^{-1} x \cdot \overline{x^{-1} g}} \mathcal{D}_{g^{-1} x \cdot y \cdot\left(\overline{y^{-1} x^{-1} g}\right)^{-1}}\right)
$$

with $g^{-1} x \cdot \overline{x^{-1} g}, g^{-1} x \cdot y \cdot\left(\overline{y^{-1} x^{-1} g}\right)^{-1} \in H$, it follows that $w^{\prime}[x, y]$ is an invertible element in $\mathcal{D}_{x} \mathcal{D}_{x y}$ for all $x, y \in G$. 
Our goal in this section is the next:

Proposition 6.3. With the above notation,

$$
\alpha^{\prime}=\left(\left\{\mathcal{D}_{x}\right\}_{x \in G},\left\{\alpha_{x}^{\prime}\right\}_{x \in G},\left\{w_{x, y}^{\prime}\right\}_{(x, y) \in G \times G}\right)
$$

is a transitive twisted partial action of $G$ on $\mathcal{A}$ with

$$
w[x, y]=\alpha_{x}\left(\varepsilon_{y} 1_{x^{-1}}\right) \varepsilon_{x} w^{\prime}[x, y] \varepsilon_{x y}^{-1}, \quad \forall x, y \in G,
$$

where $\alpha_{x}^{\prime}(a)=\varepsilon_{x}^{-1} \alpha_{x}(a) \varepsilon_{x}$ and

$$
\varepsilon_{x}=1_{x} \prod_{g \in \Lambda} \theta_{g^{-1}}\left(w\left[g^{-1}, x\right] w\left[g^{-1} x \cdot \overline{x^{-1} g},\left(\overline{x^{-1} g}\right)^{-1}\right]^{-1}\right) \in \mathcal{U}\left(\mathcal{D}_{x}\right),
$$

for all $x \in G$ and $a \in \mathcal{D}_{x^{-1}}$. In particular, $\alpha$ and $\alpha^{\prime}$ are equivalent.

Proof. First note that by Lemma 5.1 and (10),

$$
\mathcal{R}_{g} \subseteq \mathcal{D}_{x} \Longleftrightarrow \mathcal{R}_{1} \subseteq \mathcal{D}_{g^{-1} x}
$$

and since

$$
w\left[g^{-1}, x\right] \in U\left(\mathcal{D}_{g^{-1}} \mathcal{D}_{g^{-1} x}\right), w\left[g^{-1} x \cdot \overline{x^{-1} g},\left(\overline{x^{-1} g}\right)^{-1}\right] \in U\left(\mathcal{D}_{g^{-1} x \cdot \overline{x^{-1} g}} \mathcal{D}_{g^{-1} x}\right)
$$

and $g^{-1} x \cdot \overline{x^{-1} g} \in H$, it follows that $\varepsilon_{x}$ is an invertible element in $\mathcal{D}_{x}$. Thus by Lemma 6.2 $\alpha^{\prime}$ is a twisted partial action, and it is clearly transitive, so it remains to concentrate on proving (16). We have

$$
w[x, y]=1_{x} 1_{x y} \prod_{g \in \Lambda} \theta_{g^{-1}}\left(\alpha_{g^{-1}}\left(w[x, y] 1_{g}\right)\right),
$$

for any $x, y \in G$ by the definition of $\theta_{g^{-1}}$. Applying the 2-cocycle equality (14) for the triple $\left(g^{-1}, x, y\right)$, and using (11), we obtain

$$
\begin{aligned}
w[x, y]=1_{x} 1_{x y} \prod_{g \in \Lambda} \theta_{g^{-1}}( & w\left[g^{-1}, x\right] w\left[g^{-1} x, y\right] 1_{g^{-1} x \cdot \overline{x^{-1} g}} \\
& \left.\times 1_{g^{-1} x \cdot y \cdot \overline{y^{-1} x^{-1} g}} w\left[g^{-1}, x y\right]^{-1}\right),
\end{aligned}
$$

as $g^{-1} x \cdot \overline{x^{-1} g}, g^{-1} x \cdot y \cdot \overline{y^{-1} x^{-1} g} \in H$. Next, equality (14), used for the triples $\left(g^{-1} x \cdot \overline{x^{-1} g},\left(\overline{x^{-1} g}\right)^{-1}, y\right)$ and $\left(g^{-1} x \cdot \overline{x^{-1} g},\left(\overline{x^{-1} g}\right)^{-1} \cdot y \cdot \overline{y^{-1} x^{-1} g},\left(\overline{y^{-1} x^{-1} g}\right)^{-1}\right)$ respectively, gives

$$
\begin{aligned}
& w\left[g^{-1} x, y\right] 1_{g^{-1}} x \cdot \overline{x^{-1} g}=w\left[g^{-1} x \cdot \overline{x^{-1} g},\left(\overline{x^{-1} g}\right)^{-1}\right]^{-1} \\
& \quad \cdot \alpha_{g^{-1} x \cdot \overline{x^{-1} g}}\left(w\left[\left(\overline{x^{-1} g}\right)^{-1}, y\right] 1 \overline{\left(\overline{x^{-1} g}\right)^{-1} \cdot x^{-1} g}\right) w\left[g^{-1} x \cdot \overline{x^{-1} g},\left(\overline{x^{-1} g}\right)^{-1} \cdot y\right]
\end{aligned}
$$

and

$$
\begin{aligned}
& w\left[g^{-1} x \cdot \overline{x^{-1} g},\left(\overline{x^{-1} g}\right)^{-1} \cdot y\right] 1_{g^{-1} x y \cdot \overline{y^{-1} x^{-1} g}} \\
& \left.\quad=\alpha_{g^{-1} x \cdot \overline{x^{-1} g}}\left(w\left[\overline{\left(x^{-1} g\right.}\right)^{-1} \cdot y \cdot \overline{y^{-1} x^{-1} g},\left(\overline{y^{-1} x^{-1} g}\right)^{-1}\right] 1_{\left(\overline{x^{-1}}\right)^{-1} \cdot x^{-1} g}\right)^{-1} \\
& \quad w\left[g^{-1} x \cdot \overline{x^{-1} g},\left(\overline{x^{-1} g}\right)^{-1} \cdot y \cdot \overline{y^{-1} x^{-1} g}\right] w\left[g^{-1} x y \cdot \overline{y^{-1} x^{-1} g},\left(\overline{y^{-1} x^{-1} g}\right)^{-1}\right] .
\end{aligned}
$$

Making consecutive replacements in our expression of $w[x, y]$, we come to

$$
w[x, y]=\varepsilon_{x}\left(\prod_{g \in \Lambda} \theta_{g^{-1}} \circ \alpha_{g^{-1} x \cdot \overline{x^{-1} g}}\left(a 1_{\left(\overline{x^{-1} g}\right)^{-1} \cdot x^{-1} g}\right)\right) w^{\prime}[x, y] \varepsilon_{x y},
$$


where

$$
\begin{aligned}
a=\alpha_{g^{-1} x} \cdot \overline{x^{-1} g}(w & {\left[\left(\overline{x^{-1} g}\right)^{-1}, y\right], w\left[\left(\overline{x^{-1} g}\right)^{-1} \cdot y \cdot \overline{y^{-1} x^{-1} g},\left(\overline{y^{-1} x^{-1} g}\right)^{-1}\right]^{-1} } \\
& \left.\times 1_{\left(\overline{x^{-1} g}\right)^{-1} \cdot x^{-1} g}\right) \in \mathcal{D}_{\left(\overline{x^{-1} g}\right)^{-1}} \mathcal{D}_{\left(\overline{x^{-1} g}\right)^{-1}} \cdot \mathcal{D}_{\left(\overline{x^{-1} g}\right)^{-1} \cdot y \cdot \overline{y^{-1} x^{-1} g}}
\end{aligned}
$$

so that it remains to show that

$$
1_{x y} \varepsilon_{x} \prod_{g \in \Lambda} \theta_{g^{-1}} \circ \alpha_{g^{-1} x \cdot \overline{x^{-1} g}}\left(a 1_{\left(\overline{x^{-1} g}\right)^{-1} \cdot x^{-1} g}\right)=\alpha_{x}\left(\varepsilon_{y} 1_{x^{-1}}\right) \varepsilon_{x},
$$

for any $x, y \in G$.

It will be convenient to take in the above equality $g$ running over $\Lambda^{\prime}$; it does not cause any harm by the definition of $\theta_{g^{-1}}$. Since $a$ lies in $\mathcal{D}_{\left(\overline{x^{-1} g}\right)^{-1}}$ we may write

$$
a 1_{\left(\overline{x^{-1} g}\right)^{-1} \cdot x^{-1} g}=\alpha \frac{}{\left(\overline{x^{-1} g}\right)^{-1}} \circ \alpha_{\left(\overline{x^{-1} g}\right)^{-1}}\left(a 1_{\left(\overline{x^{-1} g}\right)^{-1} \cdot x^{-1} g}\right),
$$

so that when using this to the left hand side of (17) and composing $\alpha_{g^{-1}} \overline{x \cdot x^{-1} g} \circ$ $\alpha\left(\overline{x^{-1} g}\right)^{-1}$, we obtain that

$$
\begin{aligned}
& 1_{x y} \varepsilon_{x} \prod_{g \in \Lambda^{\prime}} \theta_{g^{-1}} \circ \alpha_{g^{-1} x \cdot \overline{x^{-1} g}}\left(a 1_{\left(\overline{x^{-1} g}\right)^{-1} \cdot x^{-1} g}\right)=1_{x} 1_{x y} \prod_{g \in \Lambda^{\prime}} \theta_{g^{-1}}\left(w\left[g^{-1}, x\right] \alpha_{g^{-1} x^{\circ}}\right. \\
& \left.\alpha_{\left(\overline{x^{-1} g}\right)^{-1}}\left(a 1_{\left(\overline{x^{-1} g}\right)^{-1} \cdot x^{-1} g}\right) w\left[g^{-1} x \cdot \overline{x^{-1} g},\left(\overline{x^{-1} g}\right)^{-1}\right]^{-1}\right),
\end{aligned}
$$

since $\alpha_{\left(\overline{x^{-1} g}\right)^{-1}}^{-1}\left(a 1_{\left(\overline{x^{-1} g}\right)^{-1} \cdot x^{-1} g}\right) \in \mathcal{D}_{\overline{x^{-1} g}} \mathcal{D}_{g^{-1} x}$. Next, putting $x=1$ in (i) of Lemma [5.2 and using (11), we have that the left hand side of (17) equals

$$
\begin{aligned}
& 1_{x} 1_{x y} \prod_{g \in \Lambda^{\prime}} \theta_{g^{-1}}\left(w\left[g^{-1}, x\right] \alpha_{g^{-1} x}\left(1_{x^{-1}} \alpha_{\left(\overline{x^{-1} g}\right)^{-1}}^{-1}\left(a 1_{\left(\overline{x^{-1} g}\right)^{-1} \cdot x^{-1} g}\right)\right)\right. \\
& \left.\times w\left[g^{-1} x \cdot \overline{x^{-1} g},\left(\overline{x^{-1} g}\right)^{-1}\right]^{-1}\right) \\
& =1_{x y} \prod_{g \in \Lambda^{\prime}} \theta_{g^{-1}}\left(\alpha_{g^{-1}} \circ \alpha_{x}\left(1_{x^{-1}} \alpha_{\left(\overline{x^{-1} g}\right)^{-1}}^{-1}\left(a 1 \frac{\left(\overline{x^{-1} g}\right)^{-1} \cdot x^{-1} g}{)}\right)\right) \varepsilon_{x}\right. \\
& =1_{x y} \prod_{g \in \Lambda^{\prime}} \theta_{g^{-1}}\left(\alpha_{g^{-1}} \circ \alpha_{x} \circ \alpha_{\left(\overline{x^{-1} g}\right)^{-1}}^{-1}\right. \\
& \left.\left(a 1 \frac{\left(\overline{x^{-1} g}\right)^{-1} \cdot x^{-1}}{1\left(\overline{x^{-1} g}\right)^{-1} \cdot x^{-1} g}\right)\right) \varepsilon_{x} .
\end{aligned}
$$

The latter, using both items of Lemma 5.2 , equals

$$
\begin{gathered}
1_{x y} \prod_{g \in \Lambda^{\prime}} \alpha_{x}\left(1_{x^{-1}} \theta_{\left(\overline{x^{-1} g}\right)^{-1}}^{-1}\left(a 1_{\left(\overline{x^{-1} g}\right)^{-1} \cdot x^{-1}} 1_{\left(\overline{x^{-1} g}\right)^{-1} \cdot x^{-1} g}\right)\right) \varepsilon_{x} \\
=\alpha_{x}\left(1_{x^{-1}} 1_{y} \prod_{g \in \Lambda^{\prime}} \theta_{\left(\overline{x^{-1} g}\right)^{-1}}(a)\right) \varepsilon_{x}=\alpha_{x}\left(\varepsilon_{y} 1_{x^{-1}}\right) \varepsilon_{x}
\end{gathered}
$$

as $\overline{x^{-1} g}$ runs over $\Lambda^{\prime}$.

\section{Existence OF A GLOBALIZATiON}

In this section we prove the existence of globalizations of partial actions on rings which are products of blocks. According to Theorem 4.1, it is enough to construct $\widetilde{w}[x, y]=\widetilde{w}_{x, y} \in \mathcal{U}(\mathcal{A})$ which satisfy the extended partial 2-cocycle equality (2). For this purpose, keeping the notation of the previous sections, define for every $x \in G$ the map $\tilde{\alpha}_{x}: \mathcal{A} \rightarrow \mathcal{A}$, by

$$
\tilde{\alpha}_{x}(a)=\alpha_{x}\left(a 1_{x^{-1}}\right)+1_{\mathcal{A}}-1_{x}
$$


with $a \in \mathcal{A}$, and set

$$
\widetilde{w}[x, y]=\tilde{\alpha}_{x}\left(\tilde{\varepsilon}_{y}\right) \tilde{\varepsilon}_{x} \widetilde{w^{\prime}}[x, y] \tilde{\varepsilon}_{x y}^{-1} \in \mathcal{U}(\mathcal{A}),
$$

where

$$
\tilde{\varepsilon}_{x}=\varepsilon_{x}+1_{\mathcal{A}}-1_{x} \in \mathcal{U}(\mathcal{A})
$$

and

$$
\widetilde{w^{\prime}}[x, y]=\prod_{g \in \Lambda^{\prime}} \theta_{g^{-1}}\left(w\left[g^{-1} x \cdot \overline{x^{-1} g},\left(\overline{x^{-1} g}\right)^{-1} \cdot y \cdot \overline{y^{-1} x^{-1} g}\right]\right) \in \mathcal{U}(\mathcal{A})
$$

with $x, y \in G$. We have by (11) that $1_{x} 1_{x y} \tilde{\alpha}_{x}\left(\tilde{\varepsilon}_{y}\right)=\alpha_{x}\left(1_{x^{-1}} 1_{y} \tilde{\varepsilon}_{y}\right)=\alpha_{x}\left(1_{x^{-1}} \varepsilon_{y}\right)$ and consequently

$$
1_{x} 1_{x y} \widetilde{w}[x, y]=w[x, y]
$$

for all $x, y \in G$.

We shall use the following composition law and 2-cocycle equality:

Lemma 7.1. For every $x, y, z \in G$ and $a \in \mathcal{A}$ we have

$$
\alpha_{x}\left(1_{x^{-1}} \tilde{\alpha}_{y}(a)\right)=1_{x} \widetilde{w}[x, y] \tilde{\alpha}_{x y}(a) \widetilde{w}[x, y]^{-1}
$$

and

$$
\varepsilon_{x}^{-1} \alpha_{x}\left(1_{x^{-1}} \widetilde{w^{\prime}}[y, z]\right) \varepsilon_{x} \widetilde{w^{\prime}}[x, y z]=1_{x} \widetilde{w^{\prime}}[x, y] \widetilde{w^{\prime}}[x y, z] .
$$

Proof. Notice first that multiplying the left hand side of (19) by $1_{x y}$ gives

$$
\begin{aligned}
\alpha_{x}\left(1_{x^{-1}} 1_{y} \tilde{\alpha}_{y}(a)\right) & =\alpha_{x}\left(\alpha_{y}\left(a 1_{y^{-1} x^{-1}} 1_{y^{-1}}\right)\right)=w[x, y]^{-1} \alpha_{x y}\left(a 1_{y^{-1} x^{-1}}\right)^{-1} w[x, y]^{-1} \\
& =1_{x} 1_{x y} \widetilde{w}[x, y] \tilde{\alpha}_{x y}(a) \widetilde{w}[x, y]^{-1}
\end{aligned}
$$

which is the right hand side multiplied by $1_{x y}$. On the other hand, it is directly checked that both sides multiplied by $1_{\mathcal{A}}-1_{x y}$ equal $1_{x}\left(1_{\mathcal{A}}-1_{x y}\right)$, which proves (19).

To prove (20), we first observe using (11), (i) of Lemma 5.2 and the obvious equality $\overline{t_{1} \overline{t_{2}}}=\overline{t_{1} t_{2}}\left(t_{1}, t_{2} \in G\right)$ that

$$
\alpha_{x}\left(1_{x^{-1}} \widetilde{w^{\prime}}[y, z]\right)=\prod_{g \in \Lambda^{\prime}} \alpha_{x}\left(1_{x^{-1}} \theta_{\left(\overline{x^{-1} g}\right)^{-1}}\left(1_{\left(\overline{x^{-1} g}\right)^{-1} \cdot x^{-1}} 1_{\left(\overline{x^{-1} g}\right)^{-1}} 1_{\left(\overline{x^{-1} g}\right)^{-1} x^{-1} g} a\right)\right),
$$

where

$$
a=w\left[\left(\overline{x^{-1} g}\right)^{-1} \cdot y \cdot \overline{y^{-1} x^{-1} g},\left(\overline{y^{-1} x^{-1} g}\right)^{-1} \cdot z \cdot \overline{z^{-1} y^{-1} x^{-1} g}\right],
$$

taking into account that $\overline{x^{-1} g}$ runs over $\Lambda^{\prime}$ when so too does $g$ with fixed $x \in G$. Next, by (ii) of Lemma 5.2, (11) and the composition law (iv) of Definition 2.1, we 
obtain

$$
\begin{aligned}
& \tilde{\varepsilon}_{x}^{-1} \alpha_{x}\left(1_{x^{-1}} \widetilde{w^{\prime}}[y, z]\right) \tilde{\varepsilon}_{x} \\
& =\tilde{\varepsilon}_{x}^{-1}\left(\prod_{g \in \Lambda^{\prime}} 1_{x} \theta_{g^{-1}} \circ \alpha_{g^{-1}} \circ \alpha_{x} \circ \alpha_{\left(\overline{x^{-1} g}\right)^{-1}}^{-1}\left(1_{\left(\overline{x^{-1} g}\right)^{-1} \cdot x^{-1}} 1_{\left(\overline{x^{-1} g}\right)^{-1}} 1_{\left(\overline{x^{-1} g}\right)^{-1} x^{-1} g} a\right)\right) \tilde{\varepsilon}_{x} \\
& =1_{x} \prod_{g \in \Lambda^{\prime}} \theta_{g^{-1}}\left(w\left[g^{-1} x \cdot \overline{x^{-1} g},\left(\overline{x^{-1} g}\right)^{-1}\right] \alpha_{g^{-1} x^{\circ}}\right. \\
& \left.\alpha_{\left(\overline{x^{-1} g}\right)^{-1}}^{-1}\left(1_{\left(\overline{x^{-1} g}\right)^{-1} \cdot x^{-1}} 1_{\left(\overline{x^{-1} g}\right)^{-1}} 1_{\left(\overline{x^{-1} g}\right)^{-1} x^{-1} g} a\right) w\left[g^{-1} x \cdot \overline{x^{-1} g},\left(\overline{x^{-1} g}\right)^{-1}\right]^{-1}\right) \\
& =1_{x} \prod_{g \in \Lambda^{\prime}} \theta_{g^{-1}}\left(w\left[g^{-1} x \cdot \overline{x^{-1} g},\left(\overline{x^{-1} g}\right)^{-1}\right] \alpha_{g^{-1} x \cdot \overline{x^{-1} g} \cdot\left(\overline{x^{-1} g}\right)^{-1}} \circ\right. \\
& \left.\alpha_{\left.\frac{(1}{\left(x^{-1} g\right.}\right)^{-1}}\left(1_{\left(\overline{x^{-1} g}\right)^{-1}} 1_{\left(\overline{x^{-1} g}\right)^{-1} x^{-1} g} a\right) w\left[g^{-1} x \cdot \overline{x^{-1} g},\left(\overline{x^{-1} g}\right)^{-1}\right]^{-1}\right) \\
& =1_{x} \prod_{g \in \Lambda^{\prime}} \theta_{g^{-1}}\left(\alpha_{g^{-1} x \cdot \overline{x^{-1} g}}\left(1_{\left(\overline{x^{-1} g}\right)^{-1} x^{-1} g} a\right)\right) .
\end{aligned}
$$

Now, $\theta_{g^{-1}}(b)=\theta_{g^{-1}}\left(\operatorname{pr}_{1} b\right)(b \in \mathcal{A})$, by the definition of $\theta_{g^{-1}}$, and therefore,

$\varepsilon_{x}^{-1} \alpha_{x}\left(1_{x^{-1}} \widetilde{w^{\prime}}[y, z]\right) \varepsilon_{x} \widetilde{w^{\prime}}[x, y z]=$

$1_{x} \prod_{g \in \Lambda^{\prime}} \theta_{g^{-1}}\left(\operatorname{pr}_{1}\left(\alpha_{g^{-1} x \cdot \overline{x^{-1} g}}\left(\operatorname{pr}_{1} a\right) w\left[g^{-1} x \cdot \overline{x^{-1} g},\left(\overline{x^{-1} g}\right)^{-1} \cdot y z \cdot\left(\overline{z^{-1} y^{-1} x^{-1} g}\right)^{-1}\right]\right)\right)$,

because $g^{-1} x \cdot \overline{x^{-1} g} \in H$ and $\mathcal{R}_{1} \subseteq \mathcal{D}_{\left(\overline{x^{-1} g}\right)^{-1} \cdot x^{-1} g}$. Finally, the automorphisms $\operatorname{pr}_{1} \circ \alpha_{h} \circ \operatorname{pr}_{1}, h \in H$ of $\mathcal{R}_{1}$ and the twisting $\operatorname{pr}_{1}\left(w\left[h, h^{\prime}\right]\right),(h \in H)$ form a twisted global action of $H$ on $\mathcal{R}_{1}$, and using the (global) 2-cocycle equality for the triple

$$
\left(g^{-1} x \cdot \overline{x^{-1} g},\left(\overline{x^{-1} g}\right)^{-1} \cdot y \cdot \overline{y^{-1} x^{-1} g},\left(\overline{y^{-1} x^{-1} g}\right)^{-1} \cdot z \cdot \overline{z^{-1} y^{-1} x^{-1} g}\right),
$$

in which each entry belongs to $H$, we conclude that

$$
\begin{aligned}
& \varepsilon_{x}^{-1} \alpha_{x}\left(1_{x^{-1}} \widetilde{w^{\prime}}[y, z]\right) \varepsilon_{x} \widetilde{w^{\prime}}[x, y z] \\
& =1_{x} \prod_{g \in \Lambda^{\prime}} \theta_{g^{-1}}\left(\operatorname { p r } _ { 1 } \left(w\left[g^{-1} x \cdot \overline{x^{-1} g},\left(\overline{x^{-1} g}\right)^{-1} \cdot y \cdot \overline{y^{-1} x^{-1} g}\right]\right.\right. \\
& \left.\cdot w\left[g^{-1} x \cdot y \cdot \overline{y^{-1} x^{-1} g},\left(\overline{y^{-1} x^{-1} g}\right)^{-1} \cdot z \cdot \overline{z^{-1} y^{-1} x^{-1} g}\right]\right)=1_{x} \widetilde{w^{\prime}}[x, y] \widetilde{w^{\prime}}[x y, z],
\end{aligned}
$$

as desired.

Now we state the following:

Theorem 7.2. Let $\mathcal{A}$ be a unital ring which is a (non-necessarily finite) product of indecomposable rings. A twisted partial action

$$
\alpha=\left(\left\{\mathcal{D}_{x}\right\}_{x \in G},\left\{\alpha_{x}\right\}_{x \in G},\{w[x, y]\}_{(x, y) \in G \times G}\right)
$$

of a group $G$ on $\mathcal{A}$ is globalizable exactly when each $\mathcal{D}_{x}(x \in G)$ is a unital ring.

Proof. It was already observed that the existence of a globalization implies that each $\mathcal{D}_{x},(x \in G)$ is unital. For the converse let $1_{x}$ be the unity of $\mathcal{D}_{x}$ and assume first that $\alpha$ is transitive. Keeping the above notation we show that the $\widetilde{w}[x, y]$ 's defined in (18) satisfy

$$
\alpha_{x}\left(\widetilde{w}[y, z] 1_{x^{-1}}\right) \widetilde{w}[x, y z]=1_{x} \widetilde{w}[x, y] \widetilde{w}[x y, z],
$$

for any $x, y, z \in G$. 
The left hand side of (21) equals

$$
\begin{aligned}
& \alpha_{x}\left(1_{x^{-1}} \tilde{\alpha}_{y}\left(\tilde{\varepsilon}_{z}\right) \tilde{\varepsilon}_{y} \widetilde{w^{\prime}}[y, z]\right) \alpha_{x}\left(1_{x^{-1}} \tilde{\varepsilon}_{y z}{ }^{-1}\right) 1_{x} \tilde{\alpha}_{x}\left(\tilde{\varepsilon}_{y z}\right) \tilde{\varepsilon}_{x}{\widetilde{w^{\prime}}}^{\prime}[x, y z] \tilde{\varepsilon}_{x y z}{ }^{-1} \\
& \quad=\alpha_{x}\left(1_{x^{-1}} \tilde{\alpha}_{y}\left(\tilde{\varepsilon}_{z}\right) \tilde{\varepsilon}_{y}\right) \varepsilon_{x} \varepsilon_{x}{ }^{-1} \alpha_{x}\left(1_{x^{-1}} \widetilde{w^{\prime}}[y, z]\right) \varepsilon_{x} \widetilde{w^{\prime}}[x, y z] \tilde{\varepsilon}_{x y z}{ }^{-1} \\
& \quad=\alpha_{x}\left(1_{x^{-1}} \tilde{\alpha}_{y}\left(\tilde{\varepsilon}_{z}\right)\right) \alpha_{x}\left(1_{x^{-1}} \tilde{\varepsilon}_{y}\right) \varepsilon_{x} \widetilde{w^{\prime}}[x, y] \widetilde{w^{\prime}}[x y, z] \tilde{\varepsilon}_{x y z}{ }^{-1}
\end{aligned}
$$

by using the definitions of $\tilde{\alpha}_{x}$ and $\varepsilon_{x}$, and the equality (20). Now, applying (19) and expanding $\widetilde{w}[x, y]^{-1}$ according to (18), we come to

$$
\begin{aligned}
& \widetilde{w}[x, y] \tilde{\alpha}_{x y}\left(\tilde{\varepsilon}_{z}\right) \widetilde{w}[x, y]^{-1} \alpha_{x}\left(1_{x^{-1}} \tilde{\varepsilon}_{y}\right) \varepsilon_{x} \widetilde{w^{\prime}}[x, y] \widetilde{w^{\prime}}[x y, z] \tilde{\varepsilon}_{x y z}{ }^{-1} \\
& =\widetilde{w}[x, y] \tilde{\alpha}_{x y}\left(\tilde{\varepsilon}_{z}\right) \tilde{\varepsilon}_{x y} \widetilde{w^{\prime}}[x, y]^{-1} \tilde{\varepsilon}_{x}{ }^{-1} \tilde{\alpha}_{x}\left(\tilde{\varepsilon}_{y}\right)^{-1} 1_{x} \alpha_{x}\left(1_{x^{-1}} \tilde{\varepsilon}_{y}\right) \varepsilon_{x} \widetilde{w}^{\prime}[x, y] \widetilde{w^{\prime}}[x y, z] \tilde{\varepsilon}_{x y z}{ }^{-1},
\end{aligned}
$$

which, after making the cancellations, gives

$$
1_{x} \widetilde{w}[x, y] \tilde{\alpha}_{x y}\left(\tilde{\varepsilon}_{z}\right) \tilde{\varepsilon}_{x y} \widetilde{w^{\prime}}[x y, z] \tilde{\varepsilon}_{x y z}{ }^{-1}=1_{x} \widetilde{w}[x, y] \widetilde{w}[x y, z],
$$

which is the right hand side of (21), as desired.

Finally, for a non-transitive $\alpha$ we partition the blocks of $\mathcal{A}$ into orbits, so that the construction of the $\widetilde{w}$ 's and the verification of (21) are obviously reduced to the transitive case. Our result follows now from Theorem 4.1 .

\section{UNIQUENESS}

In this section we extend Definition 6.1 permitting $G$ to act on distinct rings, and prove that any two globalizations of a twisted partial action of a group $G$ are equivalent, provided that the rings are products of blocks.

Definition 8.1. Let $\mathcal{A}_{1}$ and $\mathcal{A}_{2}$ be rings. We say that a twisted partial action

$$
\alpha_{1}=\left(\left\{\mathcal{D}_{x}^{(1)}\right\}_{x \in G},\left\{\alpha_{1, x}\right\}_{x \in G},\left\{w_{1}[x, y]\right\}_{(x, y) \in G \times G}\right)
$$

of $G$ on $\mathcal{A}_{1}$ is isomorphic to the twisted partial action

$$
\alpha_{2}=\left(\left\{\mathcal{D}_{x}^{(2)}\right\}_{x \in G},\left\{\alpha_{2, x}\right\}_{x \in G},\left\{w_{2}[x, y]\right\}_{(x, y) \in G \times G}\right)
$$

of $G$ on $\mathcal{A}_{2}$ if there exist a ring isomorphism $\phi: \mathcal{A}_{1} \rightarrow \mathcal{A}_{2}$ such that

$$
\begin{gathered}
\phi\left(\mathcal{D}_{x}^{(1)}\right)=\mathcal{D}_{x}^{(2)} \forall x \in G, \\
\phi \circ \alpha_{1, x} \circ \phi^{-1}(a)=\alpha_{2, x}(a) \quad \forall x \in G, a \in \mathcal{D}_{x^{-1}}^{(2)},
\end{gathered}
$$

and

$$
\phi_{x, y} w_{1}[x, y] \phi_{x, y}^{-1}=w_{2}[x, y] \forall x, y \in G
$$

as multipliers of $\mathcal{D}_{x}^{(2)} \mathcal{D}_{x y}^{(2)}$, where $\phi_{x, y}$ stands for the restriction of $\phi$ to $\mathcal{D}_{x}^{(1)} \mathcal{D}_{x y}^{(1)}$. Two globalizations

$$
\beta_{1}=\left(\mathcal{B}_{1},\left\{\beta_{1, x}\right\}_{x \in G},\left\{u_{1}[x, y]\right\}_{(x, y) \in G \times G)}\right.
$$

and

$$
\beta_{2}=\left(\mathcal{B}_{2},\left\{\beta_{2, x}\right\}_{x \in G},\left\{u_{2}[x, y]\right\}_{(x, y) \in G \times G)}\right.
$$

of a partial action

$$
\alpha=\left(\left\{\mathcal{D}_{x}\right\}_{x \in G},\left\{\alpha_{x}\right\}_{x \in G},\{w[x, y]\}_{(g, h) \in G \times G)}\right)
$$

of $G$ on $\mathcal{A}$ with embeddings $\varphi_{1}: \mathcal{A} \rightarrow \mathcal{B}_{1}$ and $\varphi_{2}: \mathcal{A} \rightarrow \mathcal{B}_{2}$ shall be called isomorphic if the actions $\beta_{1}$ and $\beta_{2}$ are isomorphic with the ring isomorphism $\phi: \mathcal{B}_{1} \rightarrow \mathcal{B}_{2}$, and

$$
\phi \circ \varphi_{1}=\varphi_{2} .
$$


We proceed with the next:

Lemma 8.2. Let

$$
\alpha=\left(\left\{\mathcal{D}_{x}\right\}_{x \in G},\left\{\alpha_{x}\right\}_{x \in G},\{w[x, y]\}_{(g, h) \in G \times G)}\right)
$$

be a twisted partial action of $G$ on a unital ring $\mathcal{A}$ and

$$
\begin{aligned}
& \beta_{1}=\left(\mathcal{B}_{1},\left\{\beta_{1, x}\right\}_{x \in G},\left\{u_{1}[x, y]\right\}_{(x, y) \in G \times G}\right), \\
& \beta_{2}=\left(\mathcal{B}_{2},\left\{\beta_{2, x}\right\}_{x \in G},\left\{u_{2}[x, y]\right\}_{(x, y) \in G \times G}\right)
\end{aligned}
$$

be globalizations of $\alpha$ with embeddings $\varphi_{1}: \mathcal{A} \rightarrow \mathcal{B}_{1}$ and $\varphi_{2}: \mathcal{A} \rightarrow \mathcal{B}_{2}$. If

$$
\varphi_{1}^{-1}\left(u_{1}[x, y] \varphi_{1}\left(1_{\mathcal{A}}\right)\right)=\varphi_{2}{ }^{-1}\left(u_{2}[x, y] \varphi_{2}\left(1_{\mathcal{A}}\right)\right) \forall x, y \in G,
$$

then $\beta_{1}$ and $\beta_{2}$ are isomorphic.

Proof. Write $\widetilde{w}[x, y]=\varphi_{1}^{-1}\left(u_{1}[x, y] \varphi_{1}\left(1_{\mathcal{A}}\right)\right),(x, y \in G)$. By (ii) of Definition 2.2, $\mathcal{B}_{1}=\sum_{x \in G} \beta_{1, x}\left(\varphi_{1}(\mathcal{A})\right)$ and $\mathcal{B}_{2}=\sum_{x \in G} \beta_{2, x}\left(\varphi_{2}(\mathcal{A})\right)$, and we claim that the map $\phi: \mathcal{B}_{1} \rightarrow \mathcal{B}_{2}$ additively determined by $\beta_{1, x}\left(\varphi_{1}(a)\right) \mapsto \beta_{2, x}\left(\varphi_{2}(a)\right),(a \in \mathcal{A})$, is well defined. For suppose that

$$
\sum_{i} \beta_{1, x_{i}}\left(\varphi_{1}\left(a_{i}\right)\right)=0
$$

and we want to be sure that $\sum_{i} \beta_{2, x_{i}}\left(\varphi_{2}\left(a_{i}\right)\right)=0$.

For all $y \in G$ and $a \in \mathcal{A}$ we have $\sum_{i} \beta_{1, x_{i}}\left(\varphi_{1}\left(a_{i}\right)\right) \beta_{1, y}\left(\varphi_{1}(a)\right)=0$ and applying $\beta_{y}^{-1}$ we obtain

$$
\begin{aligned}
& 0=\sum_{i} \beta_{1, y}^{-1} \circ \beta_{1, x_{i}}\left(\varphi_{1}\left(a_{i}\right)\right) \varphi_{1}(a) \\
& =\sum_{i} u_{1}\left[y^{-1}, y\right]^{-1} \beta_{1, y^{-1}} \circ \beta_{1, x_{i}}\left(\varphi_{1}\left(a_{i}\right)\right) u_{1}\left[y^{-1}, y\right] \varphi_{1}(a) \\
& =\sum_{i} u_{1}\left[y^{-1}, y\right]^{-1} u_{1}\left[y^{-1}, x_{i}\right] \beta_{1, y^{-1} x_{i}}\left(\varphi_{1}\left(a_{i}\right)\right) u_{1}\left[y^{-1}, x_{i}\right]^{-1} u_{1}\left[y^{-1}, y\right] \varphi_{1}(a) \\
& =\sum_{i} u_{1}\left[y^{-1}, y\right]^{-1} u_{1}\left[y^{-1}, x_{i}\right] \beta_{1, y^{-1} x_{i}}\left(\varphi_{1}\left(a_{i}\right)\right) \varphi_{1}\left(1_{y^{-1} x_{i}}\right) u_{1}\left[y^{-1}, x_{i}\right]^{-1} u_{1}\left[y^{-1}, y\right] \varphi_{1}(a),
\end{aligned}
$$

since by (iii) of Definition 2.2

$$
\beta_{1, y^{-1} x_{i}}\left(\varphi_{1}\left(a_{i}\right)\right) \varphi_{1}\left(1_{y^{-1} x_{i}}\right) u_{1}\left[y^{-1}, x_{i}\right]^{-1} u_{1}\left[y^{-1}, y\right] \varphi_{1}(a)
$$

is contained in

$$
\beta_{1, y^{-1} x_{i}}\left(\varphi_{1}(\mathcal{A})\right) \varphi_{1}(\mathcal{A})=\varphi_{1}\left(\mathcal{D}_{y^{-1} x_{i}}\right)=\varphi_{1}(\mathcal{A}) \varphi_{1}\left(1_{y^{-1} x_{i}}\right) .
$$

Next in view of $\varphi_{1}\left(1_{y^{-1} x_{i}}\right)=\varphi_{1}\left(\alpha_{y^{-1} x_{i}}\left(1_{x_{i}^{-1} y}\right)\right)=\beta_{1, y^{-1} x_{i}}\left(\varphi_{1}\left(1_{x_{i}^{-1} y}\right)\right)$ we have that the above sum equals

$$
\begin{gathered}
\sum_{i} u_{1}\left[y^{-1}, y\right]^{-1} u_{1}\left[y^{-1}, x_{i}\right] \beta_{1, y^{-1} x_{i}}\left(\varphi_{1}\left(a_{i} 1_{x_{i}^{-1} y}\right)\right) u_{1}\left[y^{-1}, x_{i}\right]^{-1} u_{1}\left[y^{-1}, y\right] \varphi_{1}(a) \\
=\sum_{i} \varphi_{1}\left(\widetilde{w}\left[y^{-1}, y\right]^{-1} \widetilde{w}\left[y^{-1}, x_{i}\right] \alpha_{y^{-1} x_{i}}\left(a_{i} 1_{x_{i}} y\right) \widetilde{w}\left[y^{-1}, x_{i}\right]^{-1} \widetilde{w}\left[y^{-1}, y\right] a\right),
\end{gathered}
$$

which implies

$$
\sum_{i} \widetilde{w}\left[y^{-1}, y\right]^{-1} \widetilde{w}\left[y^{-1}, x_{i}\right] \alpha_{y^{-1} x_{i}}\left(a_{i} 1_{x_{i}^{-1} y}\right) \widetilde{w}\left[y^{-1}, x_{i}\right]^{-1} \widetilde{w}\left[y^{-1}, y\right] a=0,
$$


as $\varphi_{1}$ is a monomorphism. Applying $\varphi_{2}$, we obtain by a symmetric calculation that

$$
\sum_{i} \beta_{2, x_{i}}\left(\varphi_{2}\left(a_{i}\right)\right) \beta_{2, y}\left(\varphi_{2}(a)\right)=0
$$

for all $y \in G$ and $a \in \mathcal{A}$. It follows that $\sum_{i} \beta_{2, x_{i}}\left(\varphi_{2}\left(a_{i}\right)\right) \mathcal{B}_{2}=0$. Now $\mathcal{B}_{2}$ is a sum of unital rings and therefore by Remark 2.5 of [11] it is right $s$-unital, i.e. for every $b \in \mathcal{B}_{2}$ there exists an element $e \in \mathcal{B}_{2}$ such that $b=b e$. Consequently, $\sum_{i} \beta_{2, x_{i}}\left(\varphi_{2}\left(a_{i}\right)\right)=0$, as claimed.

In a similar fashion, $\beta_{2, x}\left(\varphi_{2}(a)\right) \mapsto \beta_{1, x}\left(\varphi_{1}(a)\right),(x \in G, a \in \mathcal{A})$ also determines a well-defined map $\phi^{\prime}: \mathcal{B}_{2} \rightarrow \mathcal{B}_{1}$ and obviously, $\phi^{\prime} \circ \phi=\phi \circ \phi^{\prime}=1$. Consequently, $\phi$ is a bijection with inverse $\phi^{\prime}$.

Observe now that when proving (25) we have established that for all $x \in G, a, b \in$ $\mathcal{A}$,

$$
\beta_{1, y}^{-1} \circ \beta_{1, x}\left(\varphi_{1}(a)\right) \varphi_{1}(b)=\varphi_{1}(c)
$$

with

$$
c=\widetilde{w}\left[y^{-1}, y\right]^{-1} \widetilde{w}\left[y^{-1}, x\right] \alpha_{y^{-1} x}\left(a 1_{x^{-1} y}\right) \widetilde{w}\left[y^{-1}, x\right]^{-1} \widetilde{w}\left[y^{-1}, y\right] b,
$$

and similarly with $\beta_{2, x}$ and $\varphi_{2}$. This yields that $\phi$ maps $\beta_{1, x}\left(\varphi_{1}(a)\right) \cdot \beta_{1, y}\left(\varphi_{1}(b)\right)=$ $\beta_{1, y}\left(\beta_{1, y}^{-1} \circ \beta_{1, x}\left(\varphi_{1}(a)\right) \varphi_{1}(b)\right)=\beta_{1, y}\left(\varphi_{1}(c)\right)$ to $\beta_{2, y}\left(\varphi_{1}(c)\right)=\beta_{2, x}\left(\varphi_{2}(a)\right) \cdot \beta_{2, y}\left(\varphi_{2}(b)\right)$, so that $\phi$ preserves multiplication. Since addition is obviously also preserved, $\phi$ is a ring isomorphism.

It is immediately seen that for all $x \in G$ one has $\beta_{2, x} \circ \phi=\phi \circ \beta_{1, x}$ and $\phi \circ \varphi_{1}=\varphi_{2}$.

It remains to check that $\phi$ respects the twistings, i.e. the global version of (23). For taking arbitrary $x, y, z \in G$ and $a \in \mathcal{A}$, we have

$$
\begin{aligned}
u_{1} & {[x, y] \beta_{1, z}\left(\varphi_{1}(a)\right)=\beta_{1, z}\left(\beta_{1, z}^{-1}\left(u_{1}[x, y]\right) \varphi_{1}(a)\right) } \\
& =\beta_{1, z}\left(u_{1}\left[z^{-1}, z\right]^{-1} \beta_{1, z^{-1}}\left(u_{1}[x, y]\right) u_{1}\left[z^{-1}, z\right] \varphi_{1}(a)\right) \\
& =\beta_{1, z}\left(u_{1}\left[z^{-1}, z\right]^{-1} u_{1}\left[z^{-1}, x\right] u_{1}\left[z^{-1} x, y\right] u_{1}\left[z^{-1}, x y\right]^{-1} u_{1}\left[z^{-1}, z\right] \varphi_{1}(a)\right) \\
& =\beta_{1, z}\left(\varphi_{1}(d)\right),
\end{aligned}
$$

with

$$
d=\widetilde{w}\left[z^{-1}, z\right]^{-1} \widetilde{w}\left[z^{-1}, x\right] \widetilde{w}\left[z^{-1} x, y\right] \widetilde{w}\left[z^{-1}, x y\right]^{-1} \widetilde{w}\left[z^{-1}, z\right] a,
$$

by the global 2 -cocycle equality and (24). Consequently $\phi$ maps

$$
u_{1}[x, y] \beta_{1, z}\left(\varphi_{1}(a)\right)=\beta_{1, z}\left(\varphi_{1}(d)\right) \mapsto \beta_{2, z}\left(\varphi_{1}(d)\right)=u_{2}[x, y] \beta_{2, z}\left(\varphi_{2}(a)\right),
$$

by a similar calculation. It follows that $\phi\left(u_{1}[x, y] b\right)=u_{2}[x, y] \phi(b)$ for all $x, y \in G$ and $b \in \mathcal{B}_{1}$. Analogously, $\phi\left(b u_{1}[x, y]\right)=\phi(b) u_{2}[x, y]$ and thus

$$
\phi u_{1}[x, y] \phi^{-1}=u_{2}[x, y],
$$

for all $x, y \in G$, which is the global version of (23).

If $\mathcal{A}$ is a product of blocks, then we are able to show that any two unital globalizations of a partial action $\alpha$ of $G$ on $\mathcal{A}$ are equivalent in a certain sense, specified below. For this purpose we will show first that if $\beta$ is a globalization for $\alpha$ with $\mathcal{B}$ being the ring under the global action, then $\mathcal{B}$ is also a product of blocks, provided that $\mathcal{B}$ has $1_{\mathcal{B}}$. We first give the details for the transitive case in Lemma 8.3 below because of simplicity of the notation; the general case is obtained similarly, which we do in Proposition 8.4 .

Observe next that if $\mathcal{A}$ is a two-sided ideal in a (non-necessarily unital) ring $\mathcal{B}$ and if $\mathcal{A}$ has $1_{\mathcal{A}}$, then $1_{\mathcal{A}}$ is central in $\mathcal{B}$ and $\mathcal{A}=\mathcal{B} 1_{\mathcal{A}}$. Moreover, $\mathcal{A}$ is a direct 
summand of $\mathcal{B}$, for the complement of $\mathcal{A}$ in $\mathcal{B}$ is the annihilator of $1_{\mathcal{A}}$ in $\mathcal{B}$. It follows that each direct summand of $\mathcal{A}$ is a direct summand of $\mathcal{B}$.

Lemma 8.3. Let $\mathcal{A}$ be a unital ring which is a (finite or infinite) product of indecomposable rings and let

$$
\alpha=\left(\left\{\mathcal{D}_{x}\right\}_{x \in G},\left\{\alpha_{x}\right\}_{x \in G},\{w[x, y]\}_{(x, y) \in G \times G)}\right.
$$

be a transitive twisted partial action of $G$ on $\mathcal{A}$ (in particular, each $\mathcal{D}_{x},(x \in G)$, is a unital ring). Let $H, \Lambda$ and $\Lambda^{\prime}$ be as in Section 5, and let

$$
\beta=\left(\mathcal{B},\left\{\beta_{x}\right\}_{x \in G},\{u[x, y]\}_{(x, y) \in G \times G)}\right.
$$

be a globalization of $\alpha$, with the embedding $\varphi: \mathcal{A} \rightarrow \mathcal{B}$. Setting $\mathcal{R}_{g}=\beta_{g}\left(\varphi\left(\mathcal{R}_{1}\right)\right)$, $\left(g \in \Lambda^{\prime}\right)$, the following holds:

(i) $\beta_{x}\left(\mathcal{R}_{1}\right)=\beta_{y}\left(\mathcal{R}_{1}\right) \Longleftrightarrow x^{-1} y \in H$.

(ii) $\beta_{x}\left(\mathcal{R}_{g}\right)=\mathcal{R}_{\overline{x g}}$ for each $g \in \Lambda^{\prime}$ and $x \in G$.

(iii) There is an embedding

$$
\psi: \mathcal{B} \rightarrow \mathcal{B}^{\prime}=\prod_{g \in \Lambda^{\prime}} \mathcal{R}_{g}
$$

which forms commutative triangles with the projections $\mathcal{B}^{\prime} \rightarrow \mathcal{R}_{g}$ and $\mathcal{B} \rightarrow \mathcal{R}_{g}$ for each $g \in \Lambda^{\prime}$.

(iv) $\mathcal{B}$ has $1_{\mathcal{B}}$ if and only if $\psi$ is an isomorphism. In this case $\beta$ is transitive.

(v) If $\alpha$ is unitally globalizable, then any globalization of $\alpha$ is unital.

Proof. Assume for simplicity of notation that $\mathcal{A} \subseteq \mathcal{B}$ so that we can write $\mathcal{R}_{g}=$ $\beta_{g}\left(\mathcal{R}_{1}\right)$, with $g \in \Lambda^{\prime}$. We have that each block of $\mathcal{A}$ is a direct factor of $\mathcal{B}$, and for each $g \in \Lambda^{\prime}$ the ring $\mathcal{R}_{g}$ is an indecomposable direct factor of $\mathcal{B}$, which lies in $\mathcal{A}$ exactly when $g \in \Lambda$. Evidently $\mathcal{R}_{g} \neq \mathcal{R}_{g^{\prime}} \Longrightarrow \mathcal{R}_{g} \cap \mathcal{R}_{g^{\prime}}=0\left(g, g^{\prime} \in \Lambda^{\prime}\right)$. We readily have that

$$
H=\left\{x \in G: \beta_{x}\left(\mathcal{R}_{1}\right)=\mathcal{R}_{1}\right\} .
$$

Next, an invertible multiplier preserves an idempotent ideal, so that

$$
u[x, y] \beta_{z}\left(\mathcal{R}_{1}\right)=\beta_{z}\left(\mathcal{R}_{1}\right) u[x, y]=\beta_{z}\left(\mathcal{R}_{1}\right),
$$

for arbitrary $x, y, z \in G$. In particular, each $R_{g}\left(g \in \Lambda^{\prime}\right)$ is preserved by the $u[x, y]$ 's. Now, for $x, y \in G$ we have

$$
\beta_{x}\left(\mathcal{R}_{1}\right)=\beta_{y}\left(\mathcal{R}_{1}\right) \Longleftrightarrow \mathcal{R}_{1}=\beta_{y}^{-1} \circ \beta_{x}\left(\mathcal{R}_{1}\right),
$$

and the latter equals

$$
\begin{aligned}
& u\left[y^{-1}, y\right]^{-1} \beta_{y^{-1}} \circ \beta_{x}\left(\mathcal{R}_{1}\right) u\left[y^{-1}, y\right] \\
& \quad=u\left[y^{-1}, y\right]^{-1} u\left[y^{-1}, x\right] \beta_{y^{-1} x}\left(\mathcal{R}_{1}\right) u\left[y^{-1}, x\right]^{-1} u\left[y^{-1}, y\right]=\beta_{y^{-1} x}\left(\mathcal{R}_{1}\right),
\end{aligned}
$$

which gives (i).

Next observe that

$$
\beta_{x}\left(\mathcal{R}_{1}\right)=u\left[\bar{x}, \bar{x}^{-1} x\right]^{-1} \beta_{\bar{x}} \circ \beta_{\bar{x}^{-1} x}\left(\mathcal{R}_{1}\right) u\left[\bar{x}, \bar{x}^{-1} x\right]=\mathcal{R}_{\bar{x}},
$$

as $\bar{x}^{-1} x \in H$. Therefore,

$$
\beta_{x}\left(\mathcal{R}_{g}\right)=u[x, g] \beta_{x g}\left(\mathcal{R}_{1}\right) u[x, g]^{-1}=\mathcal{R}_{\overline{x g}},
$$

with $x \in G, g \in \Lambda^{\prime}$, proving (ii). 
It follows from (ii) that

$$
\beta_{x}(\mathcal{A})=\prod_{g \in \Lambda} \mathcal{R}_{\overline{x g}} \subseteq \mathcal{B},
$$

for any $x \in G$. Consider the direct product $\mathcal{B}^{\prime}=\prod_{g \in \Lambda^{\prime}} \mathcal{R}_{g}$. By the universal property of the product there is a unique homomorphism $\psi: \mathcal{B} \rightarrow \mathcal{B}^{\prime}$ giving commutative triangles with the projections $\mathcal{B}^{\prime} \rightarrow \mathcal{R}_{g}$ and $\mathcal{B} \rightarrow \mathcal{R}_{g}$ for each $g \in \Lambda^{\prime}$. Since $\mathcal{B}=\sum_{x \in G} \beta_{x}(\mathcal{A})$, an arbitrary $b \in \mathcal{B}$ can be written as $b=\beta_{x_{1}}\left(a_{1}\right)+\cdots+\beta_{x_{s}}\left(a_{s}\right)$ for some $x_{1}, \ldots, x_{s} \in G$ and $a_{1}, \ldots, a_{s} \in \mathcal{A}$. It is easy to see that one can choose the elements $a_{1}, \ldots, a_{s} \in \mathcal{A}$ so that if $\operatorname{pr}_{g}\left(\beta_{x_{i}}\left(a_{i}\right)\right) \neq 0$ for some $i$ and $g \in \Lambda^{\prime}$, then $\operatorname{pr}_{g}\left(\sum_{j \neq i} \beta_{x_{j}}\left(a_{j}\right)\right)=0$, where $\operatorname{pr}_{g}$ denotes the projection $\mathcal{B} \rightarrow \mathcal{R}_{g}$. It follows that $\psi$ is injective, proving (iii).

Now observe that $\mathcal{B}$ has $1_{\mathcal{B}}$ exactly when there are finitely many $x_{1}, \ldots, x_{s}$ in $G$ such that $\mathcal{B}=\sum \beta_{x_{i}}(\mathcal{A})$. Consequently, there exists a partition

$$
\Lambda^{\prime}=\Lambda_{1} \cup \Lambda_{2} \cup \ldots \cup \Lambda_{s}
$$

into disjoint subsets such that

$$
\prod_{g \in \Lambda_{i}} \mathcal{R}_{g} \subseteq \beta_{x_{i}}(\mathcal{A}), i=1, \ldots, s
$$

and

$$
\mathcal{B}=\left(\prod_{g \in \Lambda_{1}} \mathcal{R}_{g}\right) \oplus \ldots \oplus\left(\prod_{g \in \Lambda_{s}} \mathcal{R}_{g}\right) \cong \prod_{g \in \Lambda^{\prime}} \mathcal{R}_{g}
$$

This proves (iv).

Finally, if

$$
\left\{\overline{x_{i} g}: g \in \Lambda, i=1, \ldots, s\right\}=\Lambda^{\prime},
$$

for some $x_{1}, \ldots, x_{s} \in G$, then one can obtain a partition (26) with (27) so that (28) holds. Thus $\mathcal{B}$ has $1_{\mathcal{B}}$ exactly when (29) is verified. The latter condition, however, depends only on $\mathcal{A}, G$ and $\alpha$, and this proves (v).

Now we pass to the non-necessarily transitive case. Suppose that $\mathcal{A}$ is a (finite or infinite) product of blocks:

$$
\mathcal{A}=\prod_{\lambda \in \Lambda} \mathcal{R}_{\lambda}
$$

i.e. each $\mathcal{R}_{\lambda}$ is an indecomposable unital ring, and let

$$
\alpha=\left(\left\{\mathcal{D}_{x}\right\}_{x \in G},\left\{\alpha_{x}\right\}_{x \in G},\{w[x, y]\}_{(x, y) \in G \times G}\right)
$$

be a twisted partial action of $G$ on $\mathcal{A}$ such that each $\mathcal{D}_{x}(x \in G)$ is unital. If $\alpha$ is non-necessarily transitive, then for a given block $\mathcal{R}_{\lambda}$ its orbit is defined by $o_{\lambda}=\left\{\mathcal{R}_{\lambda^{\prime}}: \exists g \in G, \mathcal{R}_{\lambda} \subseteq \mathcal{D}_{g^{-1}}, \alpha_{g}\left(\mathcal{R}_{\lambda}\right)=\mathcal{R}_{\lambda^{\prime}}\right\}$. These are the block-orbits of $\mathcal{A}$ with respect to $\alpha$. Thus we partition the blocks into a disjoint union of block-orbits, and let $\Upsilon \subseteq \Lambda$ be such that $\left\{\mathcal{R}_{\mu}: \mu \in \Upsilon\right\}$ is a complete set of representatives of the block-orbits. For any $\mu \in \Upsilon$, put $\mathcal{O}_{\mu}=\prod \mathcal{R}_{\lambda}$, where $\mathcal{R}_{\lambda}$ runs over $o_{\mu}$. Then evidently

$$
\mathcal{A}=\prod_{\mu \in \Upsilon} \mathcal{O}_{\mu}
$$

The ring $\mathcal{O}_{\mu}$ will be called the orbit ideal corresponding to $\mu$. Clearly $\alpha$ restricts to a transitive twisted partial action of $G$ on each orbit ideal. 
Proposition 8.4. Let $\mathcal{A}$ be a unital ring which is a (finite or infinite) product of indecomposable rings and let $\alpha$ be a unitally globalizable partial action of $G$ on $\mathcal{A}$. If $\beta$ is an arbitrary globalization of $\alpha$ with $G$ acting (globally) on $\mathcal{B}$, then

(i) $\mathcal{B}$ has $1_{\mathcal{B}}$ and $\mathcal{B}$ is a product of blocks;

(ii) each block-orbit of $\mathcal{B}$ contains exactly one block orbit of $\mathcal{A}$ and this establishes a one-to-one correspondence between the block-orbits of $\mathcal{B}$ and those of $\mathcal{A}$;

(iii) the restriction of $\beta$ to an orbit ideal of $\mathcal{B}$ forms a globalization for $\alpha$ restricted to the corresponding orbit ideal of $\mathcal{A}$.

Proof. Write $\mathcal{A}$ as in (30) and assume the notation introduced above with respect to the orbits. Assume also for simplicity that $\mathcal{A} \subseteq \mathcal{B}$. Given $\mu \in \Upsilon$, we have a transitive twisted partial action of $G$ on $\mathcal{O}_{\mu}$ by means of $\alpha$, and let $\mathcal{R}_{\mu}, H(\mu), \Lambda(\mu), \Lambda^{\prime}(\mu)$ correspond to $\mathcal{R}_{1}, H, \Lambda$ and $\Lambda^{\prime}$, respectively, in the notation of Lemma8.3. Similarly as in the transitive case we have an embedding

$$
\psi: \mathcal{B} \rightarrow \prod_{\mu \in \Upsilon} \prod_{g \in \Lambda^{\prime}(\mu)} \mathcal{R}_{\mu, g},
$$

where $\mathcal{R}_{\mu, g}=\beta_{g}\left(\mathcal{R}_{\mu}\right)$. Set $\mathcal{O}_{\mu}^{\prime}=\sum_{x \in G} \beta_{x}\left(\mathcal{O}_{\mu}\right) \subseteq \mathcal{B}$. Clearly, if $\mu^{\prime} \in \Upsilon, \mu^{\prime} \neq \mu$, then $\mathcal{R}_{\mu^{\prime}, g} \cap \mathcal{O}_{\mu}^{\prime}=0$ for any $g \in \Lambda\left(\mu^{\prime}\right)$; in particular, $\mathcal{O}_{\mu}$ is the unique orbit ideal of $\mathcal{A}$ contained in $\mathcal{O}_{\mu}^{\prime}$. It is easily verified that $\beta$ restricts to $\mathcal{O}_{\mu}^{\prime}$ giving a globalization for $\alpha$ restricted to $\mathcal{O}_{\mu}$. Thus (iii) will follow from (i) and (ii) provided that the map $\mathcal{O}_{\mu} \mapsto \mathcal{O}_{\mu}^{\prime}$ will give the announced correspondence.

Since $\alpha$ is unitally globalizable, we obtain as in Lemma 8.3 that there exist finitely many elements $x_{1}, x_{2}, \ldots, x_{s} \in G$ such that

$$
\left\{\overline{x_{i} g}: g \in \Lambda(\mu), i=1, \ldots, s\right\}=\Lambda^{\prime}(\mu),
$$

for all $\mu \in \Upsilon$. On the other hand, if (32) holds, then we see as in Lemma 8.3 that $\psi$ is an isomorphism, and in particular, $\mathcal{B}$ has $1_{\mathcal{B}}$. Again (32) depends only on $\mathcal{A}, G$ and $\alpha$, so that any globalization of $\alpha$ is unital. This proves (i). For (ii) it only remains to note that the $\mathcal{O}_{\mu}^{\prime}$ 's $(\mu \in \Upsilon)$ are the orbit ideals of $\mathcal{B}$ with $\mathcal{O}_{\mu}^{\prime}=\prod_{g \in \Lambda^{\prime}(\mu)} \mathcal{R}_{\mu, g}$.

Before stating our main result on uniqueness, we give two more definitions.

Definition 8.5. Let

$$
\alpha_{1}=\left(\left\{\mathcal{D}_{x}^{(1)}\right\}_{x \in G},\left\{\alpha_{1, x}\right\}_{x \in G},\left\{w_{1}[x, y]\right\}_{(x, y) \in G \times G}\right)
$$

be a twisted partial action of $G$ on a ring $\mathcal{A}_{1}$ and

$$
\alpha_{2}=\left(\left\{\mathcal{D}_{x}^{(2)}\right\}_{x \in G},\left\{\alpha_{2, x}\right\}_{x \in G},\left\{w_{2}[x, y]\right\}_{(x, y) \in G \times G}\right)
$$

be a twisted partial action of $G$ on a ring $\mathcal{A}_{2}$ such that all $\mathcal{D}_{x}^{(1)}, \mathcal{D}_{x}^{(2)}(x \in G)$ are unital rings. We say that $\alpha_{1}$ is equivalent to $\alpha_{2}$ if $\alpha_{1}$ is isomorphic to a twisted partial action of $G$ on $\mathcal{A}_{2}$ which is equivalent to $\alpha_{2}$ in the sense of Definition 6.1.

The definition means that there exist a ring isomorphism $\phi: \mathcal{A}_{1} \rightarrow \mathcal{A}_{2}$ and a function

such that (22) holds,

$$
G \ni x \mapsto \varepsilon_{x} \in \mathcal{U}\left(\mathcal{D}_{x}^{(2)}\right) \subseteq \mathcal{A}_{2}
$$

$$
\phi \alpha_{1, x} \phi^{-1}(a)=\varepsilon_{x} \alpha_{2, x}(a) \varepsilon_{x}^{-1}, \forall x \in G, a \in \mathcal{D}_{x^{-1}}^{(2)},
$$

and

$$
\phi\left(w_{1}[x, y]\right)=\varepsilon_{x} \alpha_{2, x}\left(\varepsilon_{y} 1_{x^{-1}}\right) w_{2}[x, y] \varepsilon_{x y}^{-1}, \quad \forall x, y \in G .
$$


Observe that in this case the $w_{1}[x, y]$ 's are invertible elements in $\mathcal{D}_{x}^{(1)} \mathcal{D}_{x y}^{(1)}$, and $\phi_{x, y} w_{1}[x, y] \phi_{x, y}^{-1}$ used in (23) becomes $\phi\left(w_{1}[x, y]\right)$.

Definition 8.6. Two unital globalizations

$$
\beta_{1}=\left(\mathcal{B}_{1},\left\{\beta_{1, x}\right\}_{x \in G},\left\{u_{1}[x, y]\right\}_{(x, y) \in G \times G}\right)
$$

and

$$
\beta_{2}=\left(\mathcal{B}_{2},\left\{\beta_{2, x}\right\}_{x \in G},\left\{u_{2}[x, y]\right\}_{(x, y) \in G \times G)}\right.
$$

of a partial action

$$
\alpha=\left(\left\{\mathcal{D}_{x}\right\}_{x \in G},\left\{\alpha_{x}\right\}_{x \in G},\{w[x, y]\}_{(g, h) \in G \times G)}\right.
$$

of $G$ on $\mathcal{A}$ with embeddings $\varphi_{1}: \mathcal{A} \rightarrow \mathcal{B}_{1}$ and $\varphi_{2}: \mathcal{A} \rightarrow \mathcal{B}_{2}$ shall be called equivalent if $\beta_{1}$ is equivalent to $\beta_{2}$ in the sense of Definition 8.5 and, moreover,

$$
\phi \circ \varphi_{1}=\varphi_{2},
$$

where $\phi: \mathcal{B}_{1} \rightarrow \mathcal{B}_{2}$ is the involved ring isomorphism.

Theorem 8.7. Let $\mathcal{A}$ be a ring with $1_{\mathcal{A}}$ which is a (finite or infinite) product of indecomposable rings and let

$$
\alpha=\left(\left\{\mathcal{D}_{x}\right\}_{x \in G},\left\{\alpha_{x}\right\}_{x \in G},\{w[x, y]\}_{(x, y) \in G \times G}\right)
$$

be a unitally globalizable twisted partial action of $G$ on $\mathcal{A}$. Then any two globalizations of $\alpha$ are equivalent.

Proof. Let

$$
\beta_{1}=\left(\mathcal{B}_{1},\left\{\beta_{1, x}\right\}_{x \in G},\left\{u_{1}[x, y]\right\}_{(x, y) \in G \times G)}\right.
$$

and

$$
\beta_{2}=\left(\mathcal{B}_{2},\left\{\beta_{2, x}\right\}_{x \in G},\left\{u_{2}[x, y]\right\}_{(x, y) \in G \times G)}\right.
$$

be arbitrary globalizations of $\alpha$ with embeddings $\varphi_{1}: \mathcal{A} \rightarrow \mathcal{B}_{1}, \varphi_{2}: \mathcal{A} \rightarrow \mathcal{B}_{2}$. By (i) of Proposition 8.4, $\mathcal{B}_{1}$ and $\mathcal{B}_{2}$ are unital rings which are products of blocks. Then clearly each $\mathcal{B}_{i}(i=1,2)$ is the product of the block-orbits, and (ii) and (iii) of Proposition 8.4 permit us to reduce our proof to the case in which $\alpha$ is transitive. Then by Lemma $8.3 \beta_{1}$ and $\beta_{2}$ are also transitive. By the global case of Proposition 6.3 $\beta_{1}$ is equivalent to

$$
\beta_{1}{ }^{\prime}=\left(\mathcal{B}_{1},\left\{\beta_{1, x}^{\prime}\right\}_{x \in G},\left\{u_{1}^{\prime}[x, y]\right\}_{(x, y) \in G \times G}\right),
$$

and

$$
\beta_{2}^{\prime}=\left(\mathcal{B}_{2},\left\{\beta_{2, x}^{\prime}\right\}_{x \in G},\left\{u_{2}^{\prime}[x, y]\right\}_{(x, y) \in G \times G)}\right.
$$

is equivalent to $\beta_{2}$, where

$$
\begin{aligned}
u_{i}^{\prime}[x, y] & =\prod_{g \in \Lambda^{\prime}} \beta_{g^{-1}}^{-1} \circ \operatorname{pr}_{\varphi_{i}\left(\mathcal{R}_{1}\right)}\left(u_{i}\left[g^{-1} x \cdot \overline{x^{-1} g},\left(\overline{x^{-1} g}\right)^{-1} \cdot y \cdot \overline{y^{-1} x^{-1} g}\right]\right), \\
\beta_{i, x}^{\prime}(a) & =\eta_{i, x}^{-1} \beta_{i, x}(a) \eta_{i, x}, \\
\eta_{i, x} & =\prod_{g \in \Lambda^{\prime}} \beta_{g^{-1}}^{-1} \circ \operatorname{pr}_{\varphi_{i}\left(\mathcal{R}_{1}\right)}\left(u_{i}\left[g^{-1}, x\right] u_{i}\left[g^{-1} x \cdot \overline{x^{-1} g},\left(\overline{x^{-1} g}\right)^{-1}\right]^{-1}\right) \in \mathcal{U}\left(\mathcal{B}_{i}\right),
\end{aligned}
$$

with $x, y \in G, a \in \mathcal{B}_{i}$, and $\operatorname{pr}_{\varphi_{i}\left(\mathcal{R}_{1}\right)}$ denoting the projection $\mathcal{B}_{i} \rightarrow \varphi_{i}\left(\mathcal{R}_{1}\right), i=1,2$. Now, let $\alpha^{\prime}$ be the twisted partial action equivalent to $\alpha$ given by Proposition 6.3. By the definition of $H$, for any $h_{1}, h_{2} \in H$ we have that

$$
\operatorname{pr}_{\varphi_{i}\left(\mathcal{R}_{1}\right)} u_{i}\left[h_{1}, h_{2}\right]=\varphi_{i}\left(\operatorname{pr}_{\mathcal{R}_{1}} w\left[h_{1}, h_{2}\right]\right),
$$


and since $g^{-1} x \cdot \overline{x^{-1} g},\left(\overline{x^{-1} g}\right)^{-1} \cdot y \cdot \overline{y^{-1} x^{-1} g} \in H$, it follows that

$$
u_{i}^{\prime}[x, y] \varphi_{i}\left(1_{\mathcal{A}}\right)=\varphi_{i}\left(\widetilde{w^{\prime}}[x, y]\right)
$$

and consequently

with arbitrary $x, y \in G$.

$$
u_{i}^{\prime}[x, y] \varphi_{i}\left(1_{x} 1_{x y}\right)=\varphi_{i}\left(w^{\prime}[x, y]\right)
$$

Next, by (ii) of Lemma 5.1 and (10), for $g \in \Lambda^{\prime}$ and $x \in G$ one has

$$
\mathcal{R}_{g} \subseteq \mathcal{D}_{x} \Longleftrightarrow \mathcal{R}_{1} \subseteq \mathcal{D}_{g^{-1} x}
$$

Thus if $\mathcal{R}_{g} \subseteq \mathcal{D}_{x}$, then

$$
\mathcal{R}_{1} \subseteq \mathcal{D}_{g^{-1}} \mathcal{D}_{g^{-1} x} \ni w\left[g^{-1}, x\right]
$$

and

so that

$$
\mathcal{R}_{1} \subseteq \mathcal{D}_{g^{-1} x \cdot \overline{x^{-1} g}} \mathcal{D}_{g^{-1} x} \ni w\left[g^{-1} x \cdot \overline{x^{-1} g},\left(\overline{x^{-1} g}\right)^{-1}\right]
$$

and

$$
\operatorname{pr}_{\varphi_{i}\left(\mathcal{R}_{1}\right)} u\left[g^{-1}, x\right]=\varphi_{i}\left(\operatorname{pr}_{\mathcal{R}_{1}} w\left[g^{-1}, x\right]\right)
$$

$$
\operatorname{pr}_{\varphi_{i}\left(\mathcal{R}_{1}\right)} u\left[g^{-1} x \cdot \overline{x^{-1} g},\left(\overline{x^{-1} g}\right)^{-1}\right]=\varphi_{i}\left(\operatorname{pr}_{\mathcal{R}_{1}} w\left[g^{-1} x \cdot \overline{x^{-1} g},\left(\overline{x^{-1} g}\right)^{-1}\right]\right) .
$$

This yields that

$$
\eta_{i, x} \varphi_{i}\left(1_{x} 1_{x y}\right)=\varphi_{i}\left(\varepsilon_{x}\right),
$$

for any $x \in G$. Consequently,

$$
\beta_{i, x}^{\prime}\left(\varphi_{i}(a)\right)=\varphi_{i}\left(\alpha_{x}^{\prime}(a)\right),
$$

for any $a \in \mathcal{D}_{x^{-1}}$, and we conclude that each $\beta_{i}^{\prime}(i=1,2)$ is a globalization of $\alpha^{\prime}$.

Next, (35) means that (24) of Lemma 8.2 is verified, and, consequently, $\beta_{1}^{\prime}$ and $\beta_{2}^{\prime}$ are isomorphic globalizations of $\alpha^{\prime}$. It follows that $\beta_{1}$ and $\beta_{2}$ are equivalent globalizations of $\alpha$.

\section{ACKNOWLEDGMENTS}

This work was carried out during the first author's visits to the Federal University of Santa Catarina (Brazil) and the University of Murcia (Spain). He is grateful to colleagues from both universities for their warm hospitality.

\section{REFERENCES}

[1] F. Abadie, Sobre ações parcias, fibrados de Fell e grupóides, Ph.D. Thesis, Universidade de São Paulo, 1999.

[2] F. Abadie, Enveloping Actions and Takai Duality for Partial Actions, J. Funct. Analysis 197 (2003), no. 1, 14-67. MR 1957674 (2004c:46130)

[3] D. Bagio, W. Cortes, M. Ferrero, A. Paques, Actions of inverse semigroups on algebras, Commun. Algebra 35 (2007), no. 12, 3865-3874. MR2371262 (2008m:16067)

[4] D. Bagio, J. Lazzarin, A. Paques, Crossed products by twisted partial actions: Separability, semisimplicity and Frobenius properties, Commun. Algebra, to appear.

[5] W. Cortes, Partial Skew Polynomial Rings and Jacobson Rings, Preprint.

[6] W. Cortes, M. Ferrero, Partial skew polynomial rings: Prime and Maximal ideals, Commun. Algebra 35 (2007), no. 4, 1183-1200. MR2313659(2008b:16038)

[7] W. Cortes, M. Ferrero, H. Marubayashi, Partial Skew Polynomial Rings and Goldie Rings, Commun. Algebra 36 (2008), 4284-4295. MR2460417

[8] M. Dokuchaev, R. Exel, Associativity of crossed products by partial actions, enveloping actions and partial representations, Trans. Amer. Math. Soc. 357 (2005), no. 5, 1931-1952. MR2115083 (2005i:16066) 
[9] M. Dokuchaev, R. Exel, J. J. Simón, Crossed products by twisted partial actions and graded algebras, J. Algebra, 320, (2008), no. 8, 3278-3310. MR2450727

[10] M. Dokuchaev, M. Ferrero, A. Paques, Partial Actions and Galois Theory, J. Pure Appl. Algebra 208 (2007), no. 1, 77-87. MR2269829 (2007i:13008)

[11] M. Dokuchaev, A. del Río, J. J. Simón, Globalizations of partial actions on nonunital rings, Proc. Amer. Math. Soc. 135 (2007), no.2, 343-352. MR2255280(2007f:16075)

[12] R. Exel, Twisted partial actions: A classification of regular $C^{*}$-algebraic bundles, Proc. London Math. Soc. 74 (1997), no. 3, 417-443. MR1425329 (98d:46075)

[13] R. Exel, Partial Actions of Groups and Actions of Semigroups, Proc. Amer. Math. Soc. 126 (1998), no. 12, 3481-3494. MR1469405 (99b:46102)

[14] R. Exel, Hecke algebras for protonormal subgroups, J. Algebra 320 (2008), no. 5, 1771-1813. MR2437631

[15] R. Exel, M. Laca, J. Quigg, Partial dynamical systems and $C^{*}$-algebras generated by partial isometries, J. Operator Theory 47 (2002), no. 1, 169-186. MR1905819 (2003f:46108)

[16] M. Ferrero, Partial actions of groups on semiprime rings, Groups, rings and group rings, Lect. Notes Pure Appl. Math. 248, Chapman \& Hall/CRC, Boca Raton, FL, (2006), 155162. MR 2226190 (2007b:16076)

[17] M. Ferrero, J. Lazzarin, Partial actions and partial skew group rings, J. Algebra 319 (2008), no. $12,5247-5264$. MR 2423825

[18] P. A. Fillmore, A User's Guide to Operator Algebras, Wiley - Interscience, 1996. MR 1385461 (97i:46094)

[19] J. L. García, J. J. Simón, Morita equivalence for idempotent rings, J. Pure Appl. Algebra 76 (1991), no. 1, 39-56. MR1140639 (93b:16010)

[20] J. Kellendonk, M. V. Lawson, Partial actions of groups, Internat. J. Algebra Comput. 14 (2004), no. 1, 87-114. MR2041539 (2004m:20120)

[21] M. V. Lawson, Inverse semigroups. The theory of partial symmetries, World Scientific Publishing Co., Inc., River Edge, NJ, 1998. MR1694900 (2000g:20123)

[22] B. Steinberg, Partial actions of groups on cell complexes, Monatsh. Math. 138 (2003), no. 2, 159-170. MR.1964463 (2004e:57006)

Instituto de Matemática e Estatística, Universidade de São Paulo, 05508-090 São PAUlo, SP, BRAZIL

E-mail address: dokucha@ime.usp.br

Departamento de Matemática, Universidade Federal de Santa Catarina, 88040-900 FLORIANÓPOLIS, SC, BRAZIL

E-mail address: exel@mtm.ufsc.br

Departamento de Matemáticas, Universidad de Murcia, 30071 Murcia, España

E-mail address: jsimon@um.es 